\title{
Relatively hyperbolic groups, rapid decay algebras and a generalization of the Bass conjecture
}

\author{
Ronghui Ji, Crichton Ogle, and Bobby Ramsey \\ With an Appendix by Crichton Ogle
}

\begin{abstract}
By deploying dense subalgebras of $\ell^{1}(G)$ we generalize the Bass conjecture in terms of Connes' cyclic homology theory. In particular, we propose a stronger version of the $\ell^{1}$-Bass Conjecture. We prove that hyperbolic groups relative to finitely many subgroups, each of which posses the polynomial conjugacy bound property and nilpotent periodicity property, satisfy the $\ell^{1}$-Stronger-Bass Conjecture. Moreover, we determine the conjugacy bound for relatively hyperbolic groups and compute the cyclic cohomology of the $\ell^{1}$-algebra of any discrete group.
\end{abstract}

Mathematics Subject Classification (2010). 46L80, 20F65, 16S34.

Keywords. $B$-bounded cohomology, rapid decay algebras, Bass conjecture.

\section{Introduction}

Let $R$ be a discrete ring with unit. A classical construction of Hattori-Stallings ([Ha], $[\mathrm{St}]$ ) yields a homomorphism of abelian groups

$$
K_{0}^{a}(R) \stackrel{\mathrm{Tr}_{\mathrm{r}}^{\mathrm{HS}}}{\longrightarrow} \mathrm{HH}_{0}(R),
$$

where $K_{0}^{a}(R)$ denotes the zeroth algebraic $K$-group of $R$ and $\mathrm{HH}_{0}(R)=R /[R, R]$ its zeroth Hochschild homology group. Precisely, given a projective module $P$ over $R$, one chooses a free $R$-module $R^{n}$ containing $P$ as a direct summand, resulting in a map

$$
\operatorname{End}_{R}(P) \stackrel{\iota}{\hookrightarrow} \operatorname{End}_{R}\left(R^{n}\right)=M_{n, n}(R) \stackrel{\text { Trace }}{\longrightarrow} \mathrm{HH}_{0}(R) .
$$

One then verifies the equivalence class of $\operatorname{Tr}^{\mathrm{HS}}([P]):=\operatorname{Trace}\left(\iota\left(\operatorname{Id}_{P}\right)\right)$ in $\mathrm{HH}_{0}(R)$ depends only on the isomorphism class of $P$, is invariant under stabilization, and sends direct sums to sums. Hence it extends uniquely to a map on $K_{0}(R)$, as above.

Let $G$ be a discrete group, $k$ a commutative ring and $k[G]$ the group algebra of $G$ with coefficients in $k \subseteq \mathbb{C}$. Let $\langle G\rangle$ denote the set of conjugacy classes of $G$. Taking tensor products over $k, \mathrm{HH}_{0}(k[G])=\bigoplus_{x \in\langle G\rangle} \mathrm{HH}_{0}(k[G])_{x}=\bigoplus_{x \in\langle G\rangle} k$ 
decomposes as a direct sum of copies of $k$ indexed on $\langle G\rangle$. Let $\pi_{x}: \mathrm{HH}_{0}(k[G]) \rightarrow k$ be the projection which is the identity on the summand indexed by $x$ and sends the other summands to zero. Given a finitely generated projective module $P$ over $k[G]$, the $P$-rank of an element $g \in G$ is given by $r_{P}(g):=\pi_{\langle g\rangle} \operatorname{Tr}^{\mathrm{HS}}([P])$. By convention, the conjugacy classes associated to elements of finite order are referred to as elliptic, and the sum of all such the elliptic summand. In [Ba], H. Bass stated the following conjecture.

Bass Conjecture. If $P$ is a finitely generated projective module over $\mathbb{Z}[G]$, then $r_{P}(g)=0$ if $g \neq 1$.

Although still unknown in general, many partial results are known, beginning with the case $G$ a linear group, verified by Bass himself in [Ba]. For the integral group algebra, Linell has shown that $r_{P}(g)=0$ whenever $1 \neq g$ is of finite order. Also, the inclusion $\mathbb{Z} \hookrightarrow \mathbb{C}$ induces an inclusion $\mathrm{HH}_{0}(\mathbb{Z}[G])=\bigoplus_{x \in\langle G\rangle} \mathbb{Z} \hookrightarrow \mathrm{HH}_{0}(\mathbb{C}[G])=$ $\bigoplus_{x \in\langle G\rangle} \mathbb{C}\left(\right.$ where $\mathrm{HH}_{0}(\mathbb{C}[G])$ is computed with tensors over $\left.\mathbb{C}\right)$, which is the obvious inclusion on each summand. Thus the Bass conjecture is implied by the

Strong Bass Conjecture - SBC. For each non-elliptic class $x$, the image of the composition $\pi_{x} \circ \operatorname{Tr}^{\mathrm{HS}}: K_{0}^{a}(\mathbb{C}[G]) \rightarrow \mathrm{HH}_{0}(\mathbb{C}[G]) \rightarrow \mathrm{HH}_{0}(\mathbb{C}[G])_{x}$ is zero.

Henceforth all algebras and tensor products are assumed to be over $\mathbb{C}$. If $A$ is a Fréchet algebra and $A^{\delta}$ denotes $A$ with the discrete topology, then there is a commuting diagram

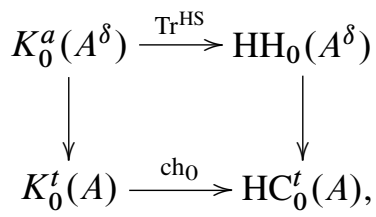

where the vertical maps are induced by the continuous map $A^{\delta} \rightarrow A$ which is the identity on elements, $\mathrm{ch}_{0}$ is the Connes-Karoubi-Chern character in dimension zero [CK], and $\mathrm{HC}_{0}^{t}(A)$ the zeroth (reduced topological) cyclic homology group of $A$. By [Ti], this same diagram exists for arbitrary topological algebras $A$ when $A$ is topologized by the fine topology, in which case one has an equality $\operatorname{HC}_{*}^{a}(A)=$ $\mathrm{HC}_{*}^{t}(A)$. The advantage to working with $\mathrm{ch}_{*}$ is that it fits into a family of Chern characters

$$
\mathrm{ch}_{n}^{m}: K_{n}^{t}(A) \rightarrow \mathrm{HC}_{n+2 m}^{t}(A)
$$

which satisfy the identity $S \circ \mathrm{ch}_{n}^{m}=\operatorname{ch}_{n}^{m-1}$ for $m \geq 1$, where $S: \operatorname{HC}_{n+2 m}^{t}(A) \rightarrow$ $\mathrm{HC}_{n+2(m-1)}^{t}(A)$ is the $S$ operator. Moreover, when $A$ is a (locally convex) algebra topologized with the fine topology, there is an isomorphism $\mathrm{HC}_{*}(A)=\mathrm{HC}_{*}^{t}(A)$, with the left-hand side denoting algebraic cyclic homology. In particular, we can take $A=\mathbb{C}[G]$ equipped with the fine topology. Then for each conjugacy class $x$, 
there is a summand $\mathrm{HC}_{*}(\mathbb{C}[G])_{x}$ of $\mathrm{HC}_{*}(\mathbb{C}[G])=\mathrm{HC}_{*}^{t}(\mathbb{C}[G])$ and a corresponding projection operator $\left(\pi_{x}\right)_{*}: \mathrm{HC}_{*}(\mathbb{C}[G]) \rightarrow \mathrm{HC}_{*}(\mathbb{C}[G])_{x}$. In light of this, it is natural to consider the following stronger version of SBC.

Stronger Bass Conjecture - SrBC. For each non-elliptic class $x$, the image of the composition $\left(\pi_{x}\right)_{*} \circ \mathrm{ch}_{*}: K_{*}^{t}(\mathbb{C}[G]) \rightarrow \mathrm{HC}_{*}(\mathbb{C}[G]) \rightarrow \mathrm{HC}_{*}(\mathbb{C}[G])_{x}$ is zero.

It follows by $[\mathrm{Og} 1]$ that the image of $\mathrm{ch}_{*}: K_{*}^{t}(\mathbb{C}[G]) \rightarrow \mathrm{HC}_{*}(\mathbb{C}[G])$ is at least the elliptic summand; the non-trivial part of the conjecture is to show that it is no more than this.

For a given non-elliptic class $x$, we say that $x$ satisfies the nilpotency condition if the operator $S^{*}: \operatorname{HC}^{*}(\mathbb{C}[G])_{x} \rightarrow \operatorname{HC}^{*+2}(\mathbb{C}[G])_{x}$ is nilpotent. We say that $G$ satisfies the nilpotency condition if $x$ does for each non-elliptic conjugacy class $x$. Combining the results of Connes-Karoubi with those of Burghelea, one has the following immediate consequence, used in [Ec1], [Ji3] and [Em]:

Observation. Let $x$ be a non-elliptic conjugacy class satisfying the nilpotency condition. Then the composition $K_{n}(\mathbb{C}[G]) \rightarrow \mathrm{HC}_{n}(\mathbb{C}[G]) \stackrel{\pi_{x}}{\longrightarrow} \mathrm{HC}_{n}(\mathbb{C}[G])_{x}$ is zero for all $n \geq 0$. Consequently, if $G$ satisfies the nilpotency condition, then the $S r B C$ is true for $G$.

Unlike SrBC, there are known examples of discrete groups which do not satisfy the nilpotency condition. However, there is a large class of groups which do. The most inclusive results are those of Emmanouil [Em]. Following [Em] we denote by $\mathcal{C}(\mathbb{C})$ the class of groups which satisfy the nilpotency condition over $\mathbb{C}$ (this is the same as over $\mathbb{Q}$, but the notation is more in keeping with the rest of our paper).

Let $\ell^{1}(G)$ denote the $\ell^{1}$-algebra of $G$. Fixing a proper word-length function $L$ on $G$, we denote by $H_{L}^{1, \infty}(G)$ the standard $\ell^{1}$-rapid-decay-algebra of $G$; this is a Fréchet subalgebra of $\ell^{1}(G)$ containing $\mathbb{C}[G]$ which is smooth in $\ell^{1}(G)$, that is, the inclusion $H_{L}^{1, \infty}(G) \hookrightarrow \ell^{1}(G)$ induces an isomorphism on topological $K$-groups in all degrees [Jo1], [Jo2]. The Connes-Karoubi-Chern character therefore produces a map

$$
K_{*}^{t}\left(\ell^{1}(G)\right) \cong K_{*}^{t}\left(H_{L}^{1, \infty}(G)\right) \rightarrow \operatorname{HC}_{*}^{t}\left(H_{L}^{1, \infty}(G)\right) .
$$

Analogous to the case of the group algebra, there is a naturally defined collection of summands $\left\{\mathrm{HH}_{*}^{t}\left(H_{L}^{1, \infty}(G)\right)_{x}\right\}$ resp. $\left\{\mathrm{HC}_{*}^{t}\left(H_{L}^{1, \infty}(G)\right)_{x}\right\}$ of $\mathrm{HH}_{*}^{t}\left(H_{L}^{1, \infty}(G)\right)$ resp. $\mathrm{HC}_{*}^{t}\left(H_{L}^{1, \infty}(G)\right)$ indexed on the set of conjugacy classes of $G$, with a similar result for topological Hochschild and cyclic cohomology. This leads to the following variant of $\mathrm{SrBC}$, which was one of our original motivations for this paper.

$\ell^{1}$-SrBC. For each non-elliptic conjugacy class $x$, the image of the composition $\left(\pi_{x}\right)_{*} \circ \mathrm{ch}_{*}: K_{*}^{t}\left(\ell^{1}(G)\right) \rightarrow \mathrm{HC}_{*}^{t}\left(H_{L}^{1, \infty}(G)\right) \rightarrow \mathrm{HC}_{*}^{t}\left(H_{L}^{1, \infty}(G)\right)_{x}$ is zero. 
It is the content of the $\ell^{1}$-Bass Conjecture that the $\ell^{1}$-Hattors-Stallings trace $\mathrm{HS}^{1}: K_{0}\left(\ell^{1}(G)\right) \rightarrow \mathrm{HH}_{0}\left(\ell^{1}(G)\right)$ has a range concentrated in elliptic summands. In [BCM] it is shown that the Baum-Connes Conjecture for a discrete group implies its $\ell^{1}$-Bass Conjecture. Thus, a-T-menable groups, in particular, amenable groups satisfy the $\ell^{1}$-Bass conjecture, following the work of Higson and Kasparov [HK]. The $\ell^{1}$-SrBC we have proposed here is stronger than the $\ell^{1}$-Bass Conjecture. Our method requires determining the nilpotency of the periodicity operator on cyclic cohomology and some geometric analysis on the group. However, it does not require the verification of Baum-Connes conjecture.

An outline of the paper is as follows. In Section 1.2, following a preliminary review of Hochschild, cyclic and periodic cyclic (co)homology, we introduce the notion of a bounding function. A family $\mathcal{B}$ of such functions, together with a wordlength function $L$ on $G$, equips $\mathbb{C}[G]$ with a collection of semi-norms, for which the completion $H_{\mathcal{B}, L}(G)$ of $\mathbb{C}[G]$ is a subalgebra of $\ell^{1}(G)$. In this paper the term rapid decay algebra refers to any subalgebra of $\ell^{1}(G)$ of this type. In fact, the rapid decay algebra $H_{L}^{1, \infty}(G)$ occurs as $H_{\mathcal{P}, L}(G)$, where $\mathcal{P}$ is the family of polynomial bounding functions. The category $\underline{B}$ of all families of bounding functions is a poset, with partial ordering given by inclusion. This category has an initial object $\mathcal{B}_{\text {min }}$, a terminal object $\mathcal{B}_{\max }$, and for all $(G, L)$ (meaning a discrete group $G$ equipped with a wordlength function $L)$ there are equalities $H_{\mathcal{B}_{\min }, L}(G)=\ell^{1}(G), H_{\mathcal{B}_{\max }, L}(G)=\mathbb{C}[G]$. Moreover, for each $(G, L)$, the association $\mathcal{B} \mapsto H_{\mathscr{B}, L}(G)$ defines a contravariant functor $F_{G, L}: \underline{\mathcal{B}} \rightarrow$ (top. alg.) such that the unique inclusion $\mathscr{B}_{\min } \succ \mathcal{B}_{\max }$ maps under $F_{G, L}$ to the natural inclusion $\mathbb{C}[G] \succ \ell^{1}(G)$. The result is a class of rapid decay algebras indexed by $\underline{B}$, lying between the group algebra $\mathbb{C}[G]$ and the $\ell^{1}$-algebra $\ell^{1}(G)$. Now there is a topological as well as a bornological approach to extending (co)homology theories (Hochschild, cyclic, periodic cyclic, bar, etc.) to non-discrete algebras. In general, these extensions apply to different categories and hence yield different theories. However, for Fréchet algebras these extensions are the same [Me2]. For $\mathcal{B}$ countable, $H_{\mathscr{B}, L}(G)$ is a Fréchet subalgebra of $\ell^{1}(G)$; consequently, on the subclass of rapid decay algebras $\left\{H_{\mathcal{B}, L}(G) \mid \mathcal{B}\right.$ countable $\}$, these two extensions agree. This is formalized in Section 1.3. Consequently, we write $F_{*}^{t}\left(H_{\mathscr{B}, L}(G)\right)$ resp. $F_{t}^{*}\left(H_{\mathcal{B}, L}(G)\right)$ to denote either topological or bornological homology resp. cohomology groups, where $F$ represents Hochschild, cyclic, periodic cyclic or bar homology. Moreover, as we further show in this section, the cohomology theories identify with the corresponding $\mathcal{B}$-bounded theory computed as the cohomology of the subcocomplex of $\mathscr{B}$-bounded cochains in the cocomplex associated to the group algebra. This idea, which is a natural extension of both [Og2] and [M1], is most conveniently cast in the context of $\mathcal{B}$-bounded cohomology theories associated to weighted simplicial sets (also defined in Section 1.3). To illustrate, there is an isomorphism

$$
\mathrm{HH}_{t}^{*}\left(H_{\mathcal{B}, L}(G)\right) \cong \mathrm{HH}_{\mathscr{B}}^{*}(\mathbb{C}[G]),
$$

where the groups on the left are the topological Hochschild cohomology groups of 
$H_{\mathcal{B}, L}(G)$, while the groups on the right are the $\mathcal{B}$-bounded Hochschild cohomology groups of $\mathbb{C}[G]$, computed as the $\mathscr{B}$-bounded cohomology of the weighted simplicial set $N_{\bullet}^{\text {cy }}(G)$, the cyclic bar construction on $G$ with weight function induced by $L$. Additionally, these groups contain summands indexed by the conjugacy classes of $G$, and which may be identified with appropriate $\mathscr{B}$-bounded cohomology groups of weighted simplicial summands of $N_{\bullet}^{\text {cy }}(G)$. This is made precise in Section 1.4. To further identify the summands in the manner suggested by Burghelea's result, we need to consider various (albeit natural) restrictions on $G$. Under the assumption that $G$ has a $\mathcal{B}$-solvable conjugacy bound there is for each conjugacy class $x \in\langle G\rangle$ an isomorphism $\mathrm{HH}_{\mathcal{B}}^{*}(\mathbb{C}[G])_{x} \cong H_{\mathcal{B}}^{*}\left(B G_{x} ; \mathbb{C}\right)$, where $G_{x}$ denotes the centralizer of $h$ in $G$ and $\langle h\rangle=x$ (Cor. 1.4.6). In the language of group theory, $\mathscr{B}$-solvability of the conjugacy bound is essentially saying that for each conjugacy class there is a bounding function $\phi \in \mathscr{B}$ for which the length of a conjugator $g$ for two conjugate elements $u$ and $v$ is bounded by $\phi$ of the lengths of $u$ and $v$. Again, for each conjugacy class $x F_{*}^{t}\left(H_{\mathcal{B}, L}(G)\right)$ contains a naturally defined summand $F_{*}^{t}\left(H_{\mathcal{B}, L}(G)\right)_{x}(F=$ $\mathrm{HH}, \mathrm{HC}, \mathrm{HPer})$, which suggests the following extension of $\ell^{1}$-SrBC.

B-SrBC. For each non-elliptic class $x$, the image of the composition

$$
\left(\pi_{x}\right)_{*} \circ \mathrm{ch}_{*}: K_{*}^{t}\left(H_{\mathcal{B}, L}(G)\right) \rightarrow \operatorname{HC}_{*}^{t}\left(H_{\mathcal{B}, L}(G)\right) \rightarrow \operatorname{HC}_{*}^{t}\left(H_{\mathcal{B}, L}(G)\right)_{x}
$$

is zero.

We say that the group $G$ satisfies the $\mathscr{B}$-nilpotency condition if the operator $S_{x}^{*}: \operatorname{HC}^{*}\left(H_{\mathcal{B}, L}(G)\right)_{x} \rightarrow \mathrm{HC}^{*+2}\left(H_{\mathcal{B}, L}(G)\right)_{x}$ is nilpotent for each non-elliptic conjugacy class $x$. As above, by Connes-Karoubi the following holds.

Observation. If $G$ satisfies the $\mathscr{B}$-nilpotency condition, then the conjecture $\mathscr{B}$-SrBC is true for $G$.

In Section 2, we use the machinery of Section 1 to verify the $\mathcal{B}$-nilpotency condition in a number of interesting cases. Although not a theorem, in practice it is generally the case that if $G$ satisfies the $\mathscr{B}$-nilpotency condition and $\mathscr{B} \subset \mathscr{B}^{\prime}$, then $G$ satisfies the $\mathscr{B}^{\prime}$-nilpotency condition. The class of groups satisfying the $\mathscr{B}$-nilpotency condition will be denoted by $\mathcal{C}_{\mathcal{B}}(\mathbb{C})$. The weakest condition is $\mathscr{B}_{\max }$-nilpotency, which is nothing else but the nilpotency condition defined above for the group algebra $\mathbb{C}[G]$ (i.e., $\mathcal{C}_{\mathcal{B}_{\max }}(\mathbb{C})=\mathcal{C}(\mathbb{C})$ ). As a warm-up, and to illustrate this method, we show in Section 2.1 that $G$ satisfies $\mathscr{B}$-nilpotency for all $\mathscr{B} \neq \mathscr{B}_{\min }$ when $G$ is (i) finitely generated nilpotent or (ii) word-hyperbolic. This includes the case $\mathscr{B}=\mathscr{P}$, which are results due to the first author [Ji1], [Ji2]. In Section 2.2 we extend this by showing

Theorem A. If $\mathcal{P} \subset \mathscr{B}$ and $G$ is hyperbolic relative to a finite set of subgroups $H_{i}$, where each $H_{i}$ is in the class $\mathcal{C}_{\mathscr{B}}(\mathbb{C})$ and has a $\mathcal{B}$-solvable conjugacy bound, then $G$ is in the class $\mathcal{C}_{\mathscr{B}}(\mathbb{C})$. In particular, if $\mathscr{B}=\mathcal{P}$, then $G$ satisfies the $\ell^{1}-S r B C$. 
Theorem A depends heavily on the following theorem on relatively hyperbolic groups.

Theorem B. Let $G$ be a finitely generated group with generating set $X$ such that $G$ is relatively hyperbolic with respect to $\left\{H_{\lambda}\right\}_{\lambda \in \Lambda}$, each member of which has $\mathcal{P}$-solvable conjugacy bound property. Then $G$ has a $\mathcal{P}$-solvable conjugacy bound.

The conjugacy problem for relatively hyperbolic groups was solved in [Bu]. However, the $\mathcal{P}$-solvable conjugacy bound property described in Theorem B is new. Recall that the idempotent conjecture for $\ell^{1}(G)$ states that the only idempotent elements in $\ell^{1}(G)$ are 0 and 1 if $G$ is torsion-free. By a standard argument we have

Corollary C. If G satisfies the hypothesis of Theorem B and is torsion-free, then the idempotent conjecture holds for $\ell^{1}(G)$.

In Section 2.3, we consider the case when $G_{x}$ is synchronously combable for non-elliptic $x$; the results of this section are preliminary, and indicate a need for further study. One important family of combable groups are those which are semihyperbolic in the sense of $[A B]$. As we have already seen, $\mathcal{B}$-solvability is needed at the non-elliptic classes in order to properly identify the resulting summands of the topological Hochschild and cyclic groups. Our second main result is

Theorem D. If $G$ is semi-hyperbolic, and $\mathcal{B}$ is any class containing $\mathcal{E}$ (the exponential bounding class), then $G$ is in $\mathrm{C}_{\mathcal{B}}(\mathbb{C})$ if it is in $\mathrm{C}_{\mathcal{B}_{\max }}(\mathbb{C})$.

For certain classes of semi-hyperbolic groups, this result can be strengthened to where $\mathcal{E}$ is replaced by $\mathcal{P}$ in the above theorem. Finally in Section 2.4 , we deal with the class $\mathscr{B}_{\min }$ (corresponding to the full $\ell^{1}$-algebra $\ell^{1}(G)$ ). In marked contrast to the structure of the topological cyclic homology groups of $H_{\mathcal{B}, L}(G)$ when $\mathcal{B} \neq \mathscr{B}_{\text {min }}$, we have

Theorem E. For each conjugacy class $x$, there is an isomorphism

$$
\mathrm{HC}_{t}^{*}\left(\ell^{1}(G)\right)_{x} \cong H_{b}^{*}\left(B G_{x} ; \mathbb{C}\right) \otimes \mathrm{HC}^{*}(\mathbb{C}),
$$

where the $S_{x}^{*}$ map on the left identifies with $\operatorname{Id} \otimes S^{*}$ on the right, and $H_{b}^{*}(\cdot)$ denotes bounded cohomology. Thus in the case of the $\ell^{1}$-algebra, the map $S_{x}^{*}$ is never nilpotent for any conjugacy class $x$. Consequently, the class $\mathrm{C}_{\mathcal{B}_{\min }}(\mathbb{C})$ is empty.

The essential reason for this result is the vanishing of bounded cohomology for amenable groups in positive degrees. It is this theorem that motivates the consideration of larger classes of bounding functions (or equivalently, the restriction to rapid decay subalgebras of $\left.\ell^{1}(G)\right)$.

Finally, it is natural to expect a connection between the conjecture $\mathcal{B}$-SrBC and the corresponding Baum-Connes conjecture for the rapid decay algebra $H_{\mathcal{B}, L}(G)$. In 
fact, the rational surjectivity of the (appropriately defined) Baum-Connes assembly map for $K_{*}^{t}\left(H_{\mathcal{B}, L}(G)\right)$ implies $\mathcal{B}$-SrBC (a very special case of this result was shown in $[\mathrm{BCM}])$. A proof of this result is provided in the appendix.

We would like to thank Denis Osin for some conversations regarding relatively hyperbolic groups and Tim Riley for his generous help on the conjugacy problems for nilpotent and other classes of groups. Some of the ideas of the paper grow out of the workshop "The property of rapid decay" in January 2006 at the American Institute of Mathematics. The first and third author wish to thank the institute for providing active and friendly atmosphere for the workshop.

\section{Hochschild and cyclic (co)homology of general rapid decay algebras}

1.1. Preliminaries. We recall the construction of Hochschild and cyclic homology ([Co1], [Lo]), assuming throughout that the base field is $\mathbb{C}$, with all algebras and tensor products being over $\mathbb{C}$. For an associative unital algebra $A$ and an $A$-bimodule $M$, let $C_{n}(A, M)=M \otimes A^{\otimes n}$. Define $b_{n}: C_{n}(A, M) \rightarrow C_{n-1}(A, M)$ by the equation

$$
\begin{aligned}
& b_{n}\left(m, a_{1}, \ldots, a_{n}\right)=( m a_{1}, a_{2}, \ldots, a_{n}+\sum_{i=1}^{n-1}(-1)^{i}\left(m, \ldots, a_{i} a_{i+1}, \ldots, a_{n}\right) \\
&+(-1)^{n}\left(a_{n} m, a_{1}, \ldots, a_{n-1}\right) .
\end{aligned}
$$

This is a differential, and the resulting complex $C_{*}(A, M)=\left(C_{*}(A, M), b\right)$ is the Hochschild complex of $A$ with coefficients on $M$. Its homology is denoted $\mathrm{HH}_{*}(A, M)$. When $M=A$, viewed in the obvious way as an $A$-bimodule, we write $C_{*}(A, A)$ simply as $C_{*}(A)$, referred to as the Hochschild complex of $A$. The complex $C_{*}(A)$ admits a degree one chain map $B_{*}$ given by

$$
\begin{aligned}
B_{n}\left(a_{0}, a_{1}, \ldots, a_{n}\right)= & \sum_{i=0}^{n}(-1)^{n i}\left(1, a_{i}, a_{i+1}, \ldots, a_{n}, a_{0}, a_{1} \ldots, a_{i-1}\right) \\
& -\sum_{i=0}^{n}(-1)^{n i}\left(a_{i}, 1, a_{i+1}, \ldots, a_{n}, a_{0}, \ldots, a_{i-1}\right) .
\end{aligned}
$$

Let $\mathscr{B}^{\prime}(A)_{* *}$ denote the first quadrant bicomplex with $\mathscr{B}^{\prime}(A)_{2 p, q}=\otimes^{q+1} A$ for $p \geq 0, \mathscr{B}^{\prime}(A)_{2 p, q}=0$ for $p<0$ and $\mathscr{B}^{\prime}(A)_{2 p-1, q}=0$ for all $p$. The differentials are $b_{q}: \mathscr{B}^{\prime}(A)_{2 p, q} \rightarrow \mathcal{B}^{\prime}(A)_{2 p, q-1}$ and $B_{q}: \mathscr{B}^{\prime}(A)_{2 p, q} \rightarrow \mathscr{B}^{\prime}(A)_{2 p-2, q+1}$ for $p \geq 1$. The associated total complex is the cyclic complex of $A$, denoted $\mathrm{CC}_{*}(A)$; because we are over a field of characteristic 0 , this is quasi-isomorphic to the smaller complex $\left(C_{*}(A) /\left(1-\tau_{*}\right), b_{*}\right)$ (which we also denote by $\mathrm{CC}_{*}(A)$ when there is no confusion), where $\tau_{n}\left(a_{0}, \ldots, a_{n}\right)=(-1)^{n}\left(a_{n}, a_{0}, \ldots, a_{n-1}\right)$. One may also form the larger 2-periodic bicomplex $\mathscr{B}(A)_{* *}$ with $\mathcal{B}(A)_{2 p, q}=$ $\bigotimes^{q+1} A, \mathscr{B}(A)_{2 p-1, q}=0$. The differentials are $b_{q}: \mathcal{B}(A)_{2 p, q} \rightarrow \mathscr{B}(A)_{2 p, q-1}$ 
and $B_{q}: \mathscr{B}(A)_{2 p, q} \rightarrow \mathscr{B}(A)_{2 p-2, q+1}$, as before. The total complex $T_{*}(A)=$ $\left(\bigoplus_{2 p+q=*} \mathscr{B}(A)_{2 p, q}, b+B\right)$ of the resulting bicomplex $\mathscr{B}(A)_{* *}=\left\{\mathscr{B}(A)_{* *} ; b, B\right\}$ is clearly $\mathbb{Z} / 2$-graded (and contractible). There is a decreasing filtration by subcomplexes $F^{k} T_{*}(A)=\left(b\left(C_{k}(A)\right) \bigoplus_{n} C_{n \geq k}(A), b+B\right)$, and the periodic cyclic complex $\mathrm{CC}_{*}^{\mathrm{per}}(A)$ is the completion of $T_{*}(A)$ with respect to this filtraton: $\mathrm{CC}_{*}^{\text {per }}(A)=$ $\lim _{n} T_{*}(A) / F^{k} T_{*}(A)$, with homology $\mathrm{HC}_{*}^{\text {per }}(A)$. The dual constructions yield Hoch$\overleftarrow{\text { schild, }}_{n}^{n}$ cyclic and periodic cyclic cohomology. Finally we recall the definition of Hochschild homology and cohomology in terms of derived functors. For a unital algebra $A, A^{\text {op }}$ denotes $A$ with the opposite multiplication. An $A$-bimodule $M$ is naturally a right $A \otimes A^{\mathrm{op}}$-module via the operation $M \otimes\left(A \otimes A^{\mathrm{op}}\right) \ni m \otimes(a \otimes b) \mapsto b m a$ (in particular, $A$, via its canonical $A$-bimodule structure, is an $A \otimes A^{\mathrm{op}}$-module). One then has $\mathrm{HH}_{*}(A, M)=\operatorname{Tor}_{*}^{A \otimes A^{\mathrm{op}}}(A, M)$, and the dual groups $\mathrm{HH}^{*}(A, M)=$ $\operatorname{Ext}_{A \otimes A^{\text {op }}}^{*}(A, M)$ defined for a left $A \otimes A^{\text {op }}$-module $M$.

Note 1.1.1. The Hochschild cohomology of $A$, denoted $\mathrm{HH}^{*}(A)$, is the cohomology of the cocomplex $\left(\operatorname{Hom}\left(C_{*}(A), \mathbb{C}\right), b^{*}\right)$. In terms of the above, this identifies with $\mathrm{HH}^{*}\left(A, A^{*}\right)$, where $A^{*}$ is the $A$-bimodule of linear functionals on $A$. Similarly, the cyclic resp. periodic cyclic cohomology of $A$ is computed as $\mathrm{HC}^{*}(A)=H^{*}\left(\mathrm{CC}^{*}(A)\right)$ resp. $\operatorname{HPer}^{*}(A)=H^{*}\left(\operatorname{CPer}^{*}(A)\right)$, where $\mathrm{CC}^{*}(A):=$ $\operatorname{Hom}\left(\mathrm{CC}_{*}(A), \mathbb{C}\right)$ resp. $\operatorname{CPer}^{*}(A):=\operatorname{Hom}\left(\mathrm{CPer}_{*}(A), \mathbb{C}\right)$.

A fundamental result and starting point for the computations in this paper is the well-known computation of the cyclic homology of $\mathbb{C}[G]$, due to Burghelea [Bur], which we summarize as follows.

Theorem 1.1.2 (Burghelea). (i) There are canonical isomorphisms

$$
\begin{aligned}
& \mathrm{HH}_{*}(\mathbb{C}[G]) \cong \bigoplus_{x \in\langle G\rangle} \mathrm{HH}_{*}(\mathbb{C}[G])_{x}, \\
& \mathrm{HC}_{*}(\mathbb{C}[G]) \cong \bigoplus_{x \in\langle G\rangle} \mathrm{HC}_{*}(\mathbb{C}[G])_{x},
\end{aligned}
$$

with the maps in the Connes-Gysin long exact sequence preserving this decomposition.

(ii) For all $x$, there is a canonical isomorphism $\mathrm{HH}_{*}(\mathbb{C}[G])_{x}=H_{*}\left(B C_{g} ; \mathbb{C}\right)$, where $C_{g}$ denotes the centralizer of $g \in G(\langle g\rangle=x)$.

(iii) If $x$ is elliptic, then $\mathrm{HC}_{*}(\mathbb{C}[G])_{x}=H_{*}\left(B C_{g} ; \mathbb{C}\right) \otimes \mathrm{HC}_{*}(\mathbb{C})$, where $S_{x}: \mathrm{HC}_{*}(\mathbb{C}[G])_{x} \rightarrow \mathrm{HC}_{*-2}(\mathbb{C}[G])_{x}$ is given by $S_{x}=\mathrm{Id} \otimes S_{*}^{\mathbb{C}}, S_{*}^{\mathbb{C}}: \mathrm{HC}_{*}(\mathbb{C}) \rightarrow$ $\mathrm{HC}_{*-2}(\mathbb{C})$.

(iv) If $x$ is non-elliptic, then $\mathrm{HC}_{*}(\mathbb{C}[G])_{x}=H_{*}\left(B N_{g} ; \mathbb{C}\right)$, where $N_{g}=C_{g} /(g)$ is the quotient of $C_{g}$ by the infinite cyclic subgroup generated by $g$. Under this identification, the summand of the Connes-Gysin sequence indexed by $x$ identifies with the Gysin sequence in homology (with coefficients in $\mathbb{C}$ ) associated to the fibration $S^{1}=\mathbb{Z} \rightarrow B C_{g} \rightarrow B N_{g}$. 
(v) The analogues of (i)-(iv) hold for the Hochschild and cyclic cohomology of $\mathbb{C}[G]$ (with direct sum replaced by direct product in (i)).

\subsection{Bounding functions and rapid decay algebras. We begin with}

Definition 1.2.1. A bounding class $\mathcal{B}$ is a non-empty set of non-decreasing functions $\left\{f: \mathbb{R}_{+} \rightarrow \mathbb{R}^{+}\right\}$satisfying the following conditions:

(i) It is closed under the operation of taking positive rational linear combinations.

(ii) If $f_{1}, f_{2} \in \mathcal{B}$, there is an $f_{3} \in \mathcal{B}$ such that $f_{1} \circ f_{2} \leq f_{3}$.

(iii) It contains the constant function 1.

(iv) If $f_{1} \in \mathscr{B}$ and $g$ is a non-decreasing linear function on $\mathbb{R}_{+}$, then there is an $f_{2} \in \mathcal{B}$ with $f_{1} \circ g \leq f_{2}$.

By (1.2.1) (iii) The smallest bounding class is $\mathscr{B}_{\min }=\mathbb{Q}^{+}$, the set of constant functions with value a positive rational number. There is also a largest class $\mathcal{B}_{\max }=$ $\left\{f: \mathbb{R}_{+} \rightarrow \mathbb{R}^{+} \mid f\right.$ is non-decreasing $\}$. Other basic classes that will appear in this paper are $\mathscr{L}:=\left\{a+b x \mid a, b \in \mathbb{Q}^{+}\right\}, \mathcal{P}:=$ the set of positive rational linear combinations of $\left\{(1+x)^{m}\right\}_{m \in \mathbb{N}}$, and $\mathcal{E}:=$ the closure, under composition, of the set of positive rational linear combinations of $\left\{C^{x}\right\}_{C \in \mathbb{Q}, C \geq 1}$ (note: if $\mathscr{L} \subseteq \mathscr{B}$, then condition (ii) implies condition (iv)). Two bounding classes $\mathscr{B}$ and $\mathscr{B}^{\prime}$ are equivalent, written $\mathcal{B} \simeq \mathcal{B}^{\prime}$, if for each $f$ in one class, there is an $f^{\prime}$ in the other with $f \leq f^{\prime}$. For two bounding classes $\mathscr{B}, \mathscr{B}^{\prime}$ we write $\mathcal{B}+\mathscr{B}^{\prime}$ to indicate the bounding class generated by $\mathscr{B} \cup \mathscr{B}^{\prime}$; this is the smallest bounding class containing both $\mathscr{B}$ and $\mathscr{B}^{\prime}$. If $\mathcal{B} \simeq \mathscr{B}+\mathcal{B}^{\prime}$, we write $\mathcal{B}^{\prime} \preceq \mathcal{B}$, and $\mathcal{B}^{\prime} \prec \mathscr{B}$ if $\mathcal{B}^{\prime} \preceq \mathscr{B}$ but $\mathscr{B} \npreceq \mathscr{B}^{\prime}$. Thus $\mathcal{B}_{\min } \prec \mathscr{L} \prec \mathcal{P} \prec \mathcal{E} \prec \mathcal{B}_{\max }$.

A weighted set $(X, w)$ will refer to a countable discrete set $X$ together with a proper function $w: X \rightarrow \mathbb{R}_{+}$. Let $C_{c}(X)$ denote the space of complex-valued functions on $X$ with compact (i.e., finite) support. A morphism $\phi:(X, w) \rightarrow\left(X^{\prime}, w^{\prime}\right)$ is $\mathcal{B}$ bounded if

for all $f \in \mathscr{B}$ there exists $f^{\prime} \in \mathcal{B}$ with $f\left(w^{\prime}(\phi(x))\right) \leq f^{\prime}(w(x))$ for all $x \in X$.

Given a weighted set $(X, w)$ and a bounding class $\mathcal{B}$, one may form the topological space

$$
H_{\mathscr{B}, w}(X):=\left\{\phi: X \rightarrow \mathbb{C}|\phi|_{f}:=\sum_{x \in X}|\phi(x)| f(w(x))<\infty \text { for all } f \in \mathcal{B}\right\} .
$$

A $\mathscr{B}$-isomorphism $\phi:(X, w) \rightarrow\left(X^{\prime}, w^{\prime}\right)$ is an isomorphism of sets which is $\mathcal{B}$ bounded, and which admits a two-sided inverse that is also $\mathscr{B}$-bounded. There is a natural embedding $H_{\mathscr{B}, w}(X) \hookrightarrow \ell^{1}(X)$, and so we will typically represent elements of $H_{\mathcal{B}, w}(X)$ in terms of their corresponding (infinite) sums: $H_{\mathcal{B}, w}(X) \ni \phi$ if and only if $\sum_{x \in X} \phi(x) x$. 
In most applications below $\mathscr{B}$ is countable, in which case $H_{\mathcal{B}, w}(X)$ is a Fréchet space which alternatively may be viewed as the completion of $C_{c}(X)$ with respect to the collection of semi-norms $\left\{|\cdot|_{f}\right\}_{f \in \mathscr{B}}$.

Proposition 1.2.3. Let $(X, w),\left(X^{\prime}, w^{\prime}\right)$ be weighted sets, $\mathcal{B}, \mathfrak{B}^{\prime}$ bounding classes and $(G, L)$ a discrete group with proper word-length function.

(i) $H_{\mathcal{B}_{\min }, w}(X)=\ell^{1}(X)$; in particular, $H_{\mathcal{B}_{\min }, L}(G)=\ell^{1}(G)$, the $\ell^{1}$-algebra of $G$.

(ii) $H_{\mathcal{B}_{\max }, w}(X)=C_{c}(X)$; in particular, $H_{\mathcal{B}_{\max }, L}(G)=\mathbb{C}[G]$, the complex group algebra of $G$.

(iii) If $\mathcal{B} \simeq \mathscr{B}^{\prime}$ then $H_{\mathcal{B}, w}(X)=H_{\mathcal{B}^{\prime}, w}(X)$.

(iv) If $\mathcal{B}^{\prime} \prec \mathcal{B}$ then there is a natural inclusion $H_{\mathcal{B}, w}(X) \subseteq H_{\mathcal{B}^{\prime}, w}(X)$.

(v) If $\phi:(X, w) \rightarrow\left(X^{\prime}, w^{\prime}\right)$ is $\mathcal{B}$-bounded, it induces a continuous map of topological vector spaces $\phi: H_{\mathcal{B}, w}(X) \rightarrow H_{\mathcal{B}, w^{\prime}}\left(X^{\prime}\right)$; if $\phi$ is a $\mathcal{B}$-isomorphism, this induced map is a continuous isomorphism of topological vector spaces.

(vi) $H_{\mathcal{B}, w}(X) \cap H_{\mathcal{B}^{\prime}, w}(X)=H_{\mathscr{B}+\mathscr{B}^{\prime}, w}(X)$.

(vii) For all $(G, L)$, there is an equality $H_{\mathcal{P}, w}(G)=H_{L}^{1, \infty}(G)$, the $\ell^{1}$-Schwartz algebra of $G$. (This algebra is the completion of $\mathbb{C}[G]$ in the seminorms $\left\{v_{k}\right\}_{k \in \mathbb{N}}$ where $\left.v_{k}(\lambda):=\sum_{g \in G}|\lambda(g)|(1+L(g))^{k} ; c f .[\mathrm{Jo} 1],[\mathrm{Jo} 2]\right)$.

(viii) $H_{\mathscr{B}, L}(G)$ is a subalgebra of $\ell^{1}(G)$ for all $(G, L)$ and bounding class $\mathcal{B}$.

Proof. The only point that is not immediate is (viii). To verify this last property, it suffices to show $H_{\mathcal{B}, L}(G)$ is closed under multiplication. Given $f \in \mathcal{B}$, we have

$$
\begin{aligned}
& \left|\left(\sum \lambda_{g_{1}} g_{1}\right)\left(\sum \lambda_{g_{2}} g_{2}\right)\right|_{f} \\
& \quad=\sum_{g}\left|\sum_{g_{1} g_{2}=g} \lambda_{g_{1}} \lambda_{g_{2}}\right| f(L(g)) \\
& \quad \leq \sum_{g}\left(\sum_{g_{1} g_{2}=g}\left|\lambda_{g_{1}} \lambda_{g_{2}}\right| f\left(L\left(g_{1}\right)+L\left(g_{2}\right)\right)\right) \\
& \quad \leq \sum_{g}\left(\sum_{\substack{g_{1} g_{2}=g \\
L\left(g_{1}\right) \leq L\left(g_{2}\right)}}\left|\lambda_{g_{1}} \lambda_{g_{2}}\right| f\left(2 L\left(g_{2}\right)\right)\right)+\sum_{g}\left(\sum_{\substack{g_{1} \\
L\left(g_{2}\right) \leq L\left(g_{1}\right)}}\left|\lambda_{g_{1}} \lambda_{g_{2}}\right| f\left(2 L\left(g_{1}\right)\right)\right) \\
& \quad \leq\left|\sum \lambda_{g_{1}} g_{1}\right|_{1}\left|\sum \lambda_{g_{2}} g_{2}\right|_{f_{2}}+\left|\sum \lambda_{g_{1}} g_{1}\right|_{f_{2}}\left|\sum \lambda_{g_{2}} g_{2}\right|_{1}<\infty,
\end{aligned}
$$

where $\left|\sum \lambda_{g} g\right|_{1}$ is the $\ell^{1}$-norm and $f_{2}$ denotes any element of $\mathscr{B}$ satisfying $f(2 x) \leq$ $f_{2}(x)$ for all $x$ (this element exists by (1.2.1) (iv)).

Note. The term rapid decay subalgebra of $\ell^{1}(G)$ conventionally refers to the Schwartz algebra $H_{L}^{1, \infty}(G)$. For this paper, a rapid decay subalgebra of $\ell^{1}(G)$ will mean any one of the subalgebras $H_{\mathscr{B}, L}(G)$ associated to a bounding class $\mathcal{B}$ and a group with word-length $(G, L)$. 
Suppose now that $(X, w)$ is a weighted set and let $\lambda: X \rightarrow \mathbb{C}$. We call $f$ a bounding function for $\lambda$ if $|\lambda(x)| \leq f(w(x))$ for each $x \in X$. We set

$$
\operatorname{Hom}_{\mathcal{B}}(X, \mathbb{C})=\{\lambda: \mathscr{B} \rightarrow \mathbb{C} \mid \lambda \text { has a bounding function in } \mathscr{B}\} .
$$

Note that property (1.2.1) (i) implies $\operatorname{Hom}_{\mathscr{B}}(X, \mathbb{C})$ is a vector space over $\mathbb{C}$. Further, given $\lambda \in \operatorname{Hom}_{\mathcal{B}}(X, \mathbb{C})$ and $\phi \in H_{\mathcal{B}, w}(X)$, the series $\lambda^{*}(\phi):=\sum_{x \in X} \lambda(x) \phi(x)$ is absolutely convergent, as $\sum_{x \in X}|\lambda(x) \phi(x)| \leq|\phi|_{f}$ whenever $f$ is a bounding function for $\lambda$. It is easy to see from this last inequality that $\lambda^{*}$ so defined yields a continuous linear functional on $H_{\mathscr{B}, w}(X)$. Alternatively, the association $\lambda \mapsto \lambda^{*}$ yields a natural linear transformation $\operatorname{Hom}_{\mathcal{B}}(X, \mathbb{C}) \rightarrow H_{\mathcal{B}, w}(X)$ which is clearly injective. What is not so clear is whether it is surjective. This leads us to

Lemma 1.2.4. Let $(X, w)$ be as above, with $w$ a proper weight function, and $\mathcal{B}$ a countable bounding set. Let $H_{\mathscr{B}, w}(X)^{*}$ denote the space of continuous linear functionals on $H_{\mathscr{B}, w}(X)$, and $\operatorname{Hom}_{\mathscr{B}}(X, \mathbb{C})$ the vector space of $\mathcal{B}$-bounded functions $f: X \rightarrow \mathbb{C}$. Then the natural inclusion $\operatorname{Hom}_{\mathcal{B}}(X, \mathbb{C}) \hookrightarrow H_{\mathcal{B}, w}(X)^{*}$ is an isomorphism of vector spaces.

Proof. As $\mathcal{B}$ is countable, we can assume there exists a sequence $\left\{f_{k} \in \mathcal{B}\right\}$ satisfying the following properties:

(i) for any $f \in \mathscr{B}$ there exists $k$ with $f \leq f_{k}$,

(ii) $2 f_{k} \leq f_{k+1}$ for each $k \geq 1$.

Now there is an obvious inclusion of vector spaces. Suppose that this inclusion is not also a surjection. Then there exists $\phi \in H_{\mathscr{B}, w}(X)^{*}$ such that for each $k \geq 1$ there is an $x_{k} \in X$ with $\left|\phi\left(x_{k}\right)\right| \geq f_{k}\left(w\left(x_{k}\right)\right)$. Because $w$ is proper, we may additionally assume that $w\left(x_{k+1}\right)>w\left(x_{k}\right)$ for each $k$. Fix such a choice of $x_{k}$ for each positive integer $k$. Let $u_{k}=\phi\left(x_{k}\right) /\left|\phi\left(x_{k}\right)\right|$. Also for each $k$ choose $\alpha_{k}$ with $\alpha_{k} /\left|\alpha_{k}\right|=\overline{u_{k}}$ and $\left|\alpha_{k}\right|=f_{k}\left(w\left(x_{k}\right)\right)^{-1}$. Then

$$
\left|\sum_{k} \alpha_{k} x_{k}\right|_{f_{n}} \leq A_{n}+\sum_{k>n}\left|\alpha_{k}\right|\left|x_{k}\right|_{f_{n}} \leq A_{n}+\sum_{m \geq 1} 2^{-m}<\infty
$$

for all $n \geq 0$, which by property (i) implies $\sum_{k} \alpha_{k} x_{k} \in H_{\mathcal{B}, w}(X)$. On the other hand,

$$
\left|\phi\left(\sum_{k} \alpha_{k} x_{k}\right)\right|=\sum_{k}\left|\alpha_{k}\right|\left|\phi\left(x_{k}\right)\right| \geq \sum_{k} 1=\infty,
$$

which is a contradiction. Hence no such $\phi$ can exist.

1.3. Topological vers. bornological algebras, and weighted simplicial sets. In this section we assume that $\mathcal{B}$ is countable. There are a priori different definitions of the topological (bornological) Hochschild and cyclic homology and cohomology groups associated to the rapid decay algebras $H_{\mathscr{B}, L}(G)$. We give a brief description of each, and then show their equivalence. As we have observed above, $H_{\mathcal{B}, L}(G)$ 
is a Fréchet algebra, hence a complete, locally convex topological algebra. To do homological algebra in this setting, we recall some basic definitions.

A topological $A$-module is a complete locally convex space, together with a jointly continuous $A$-module structure. A topological $A$-module is said to be topologically projective if it is a direct summand of a topological $A$-module of the form $A \hat{\otimes} E$, for $E$ a complete locally convex space, and where $\hat{\otimes}$ is the complete projective topological tensor product.

Let $M$ be a topological $A$-module. The proper framework for defining topological homology and cohomology groups is that of relative homological algebra, where continuous surjections of topological $A$-modules are required to be $\mathbb{C}$-split [T1], $\S 2$. In this setting, a topological projective resolution of $M$ is an exact sequence of topological $A$-modules

$$
0 \leftarrow M \stackrel{\varepsilon}{\leftarrow} M_{0} \stackrel{\partial_{1}}{\longleftarrow} M_{1} \stackrel{\partial_{2}}{\longleftarrow} M_{2} \stackrel{\partial_{3}}{\longleftarrow} \ldots
$$

such that each $M_{i}$ is topologically projective, all of the maps are continuous $A$-module maps, and the resolution has a continuous linear homotopy $s_{i}: M_{i} \rightarrow M_{i+1}$ such that $\partial_{i+1} s_{i}+s_{i-1} \partial_{i}=\mathrm{id}_{M_{i}}$.

Given a topological projective resolution $P$. of $M$ and any topological $A$-module $N$, one can form the complexes

$$
P . \hat{\otimes}_{A} N=0 \leftarrow M_{0} \hat{\otimes}_{A} N \stackrel{\partial_{1} \otimes \mathrm{Id}}{\longleftarrow} M_{1} \hat{\otimes}_{A} N \stackrel{\partial_{2} \otimes \mathrm{Id}}{\longleftarrow} M_{2} \hat{\otimes}_{A} N \stackrel{\partial_{3} \otimes \mathrm{Id}}{\longleftarrow} \cdots,
$$

$\operatorname{Hom}_{A}\left(P_{\bullet}, N\right)=0 \rightarrow \operatorname{Hom}_{A}\left(M_{0}, N\right) \stackrel{\partial_{1}^{*}}{\longrightarrow} \operatorname{Hom}_{A}\left(M_{1}, N\right) \stackrel{\partial_{2}^{*}}{\longrightarrow} \operatorname{Hom}_{A}\left(M_{2}, N\right) \stackrel{\partial_{3}^{*}}{\longrightarrow} \cdots$

where $\operatorname{Hom}_{A}\left(M^{\prime}, M^{\prime \prime}\right)$ denotes the internal Hom-functor evaluated on $\left(M^{\prime}, M^{\prime \prime}\right)$ (i.e., the vector space of continuous $A$-linear maps from $M^{\prime}$ to $\left.M^{\prime \prime}\right)$. As in the algebraic case, it follows that $\operatorname{Tor}_{*}^{A}(M, N):=H_{*}\left(P . \hat{\otimes}_{A} N\right)$ and $\operatorname{Ext}_{A}^{*}(M, N):=$ $H^{*}\left(\operatorname{Hom}_{A}\left(P_{\bullet}, N\right)\right)$. These definitions are independent of the particular topological projective resolution by the usual arguments [Ta].

Note 1.3.2. Given a topological chain complex $\left(C_{*}, d_{*}\right)$ with continuous boundary maps, the unreduced $n$-th homology group is meant to mean $\operatorname{ker}\left(d_{n}\right) / \operatorname{im}\left(d_{n+1}\right)$, while the reduced $n$-th homology group is computed as $\operatorname{ker}\left(d_{n}\right) / \overline{\operatorname{im}\left(d_{n+1}\right)}$, where $\overline{\operatorname{im}\left(d_{n+1}\right)}$ denotes the closure of $\operatorname{im}\left(d_{n+1}\right)$ in $C_{n}$. The unreduced groups are "algebraically correct", while the reduced groups are "topologically correct" in the sense that the induced subquotient topology is Hausdorff if it was to begin with on the chain level. In this paper, homology groups of topological chain complexes will always be unreduced, and the homology groups viewed simply as vector spaces without any inherited topology. The same remarks apply to topological cochain complexes and cohomology. We will return to this point briefly later on.

Given a complete, locally convex topological algebra $A$ with unit and a topological $A$-bimodule $M$, one may define the topological Hochschild homology of $A$ with 
coefficients in $M$ as $\mathrm{HH}_{*}^{t}(A, M):=\operatorname{Tor}_{*}^{A \hat{\otimes}} A^{\mathrm{op}}(A, M)$. These derived functors may alternatively be computed as the homology of the topological Hochschild complex $\left(C_{*}^{t}(A, M), b_{*}\right)$ where $C_{n}(A, M):=M \widehat{\otimes} A^{\hat{\otimes} n}$, with $b_{n}$ given as above in (1.1). When $M=A$, the topological cyclic and periodic cyclic complexes are defined similarly, by taking the algebraic definition in (1.1) and replacing each occurrence of $\left(C_{*}(A, A), b_{*}\right)$ with $\left(C_{*}^{t}(A, A), b_{*}\right)$. For cohomology, one defines $\operatorname{HH}_{t}^{*}(A, M)$ as $\operatorname{Ext}_{A \hat{\otimes} A^{\text {op }}}^{*}(A, M)$. Again, by convention, $\mathrm{HH}_{t}^{*}(A)$, the topological Hochschild cohomology of $A$ which is dual to $\mathrm{HH}_{*}^{t}(A)$, may be computed as the cohomology of $\operatorname{Hom}\left(C_{*}^{t}(A), \mathbb{C}\right)$, the cocomplex of continuous functionals on $C_{*}^{t}(A)$, which in turn identifies with the cocomplex $C_{t}^{*}\left(A, A^{*}\right)\left(A^{*}\right.$ is the continuous dual of $A$; cf. (1.1.1), [Co1]). Similarly, $\operatorname{HC}_{t}^{*}(A)$ resp. $\operatorname{HPer}_{t}^{*}(A)$ is computed as the cohomology of the cocomplex $\operatorname{Hom}\left(\mathrm{CC}_{*}^{t}(A), \mathbb{C}\right)$ resp. $\operatorname{Hom}\left(\operatorname{CPer}_{*}^{t}(A), \mathbb{C}\right)$.

An alternative (and sometimes preferable) setting for doing homological algebra is the category of bornological algebras and vector spaces, as shown by Meyer in [Me2]. Briefly, a bornology on a vector space consists of a collection of bounded subsets. The bornology is convex if every bounded subset is contained in an absolutely convex bounded subset, and complete if every bounded subset is contained in a complete disk. Bornologies are assumed to be complete and convex (the original reference for these definitions are [HN1], [HN2], but [M2, §2] will be sufficient for our purposes). As in the topological case, resolutions in this category are assumed to be $\mathbb{C}$-split; moreover the (projective) complete tensor product of two bornological vector spaces $V$ and $W$ exists and is also denoted $V \hat{\otimes} W$ when there is no confusion. Working in the category of bornological unital algebras and bornological bi-modules over those algebras, one may proceed as above and define bornological Hochschild, cyclic and periodic cyclic (co)homology in terms of the appropriate derived functors. The corresponding groups will be indicated with a subscript or superscript "bor" in place of " $\mathrm{t}$ ". As these functors are originating in different categories, they are different functors. However, our primary interest will be the rapid decay algebras defined in (1.1). These are Fréchet algebras which can be viewed either topologically or bornologically.

Lemma 1.3.3. For all $(G, L)$ and countable $\mathcal{B}$, there are natural isomorphisms

$$
\begin{aligned}
F_{*}^{t}\left(H_{\mathcal{B}, L}(G)\right) \cong F_{*}^{\text {bor }}\left(H_{\mathcal{B}, L}(G)\right), \\
F_{t}^{*}\left(H_{\mathcal{B}, L}(G)\right) \cong F_{\text {bor }}^{*}\left(H_{\mathscr{B}, L}(G)\right)
\end{aligned}
$$

for $F=\mathrm{HH}$, HC or HPer.

Proof. Consider two weighted sets $(X, w)$ and $\left(X^{\prime}, w^{\prime}\right)$. As above, we may associate to these respectively the Fréchet spaces $H_{\mathcal{B}, w}(X)$ and $H_{\mathcal{B}, w^{\prime}}\left(X^{\prime}\right)$. It is then easy to see that in both the topological and bornological categories there is an isomorphism of topological resp. bornological vector spaces

$$
H_{\mathcal{B}, w}(X) \hat{\otimes} H_{\mathcal{B}, w^{\prime}}\left(X^{\prime}\right) \cong H_{\mathcal{B}, w \times w^{\prime}}\left(X \times X^{\prime}\right),
$$


where $\left(w \times w^{\prime}\right)\left(x, x^{\prime}\right)=w(x)+w^{\prime}\left(x^{\prime}\right)$. Iterating this isomorphism yields isomorphisms

$$
H_{\mathcal{B}, w}(X)^{\widehat{\otimes} m} \cong H_{\mathcal{B}, w^{m}}\left(X^{m}\right)
$$

which apply in either category (where $w^{m}=w \times w \times \cdots \times w, m$ times). Specializing to the case $(X, w)=(G, L)$, we see that for both projective complete tensor products there is an isomorphism

$$
H_{\mathcal{B}, L}(G)^{\widehat{\otimes} m} \cong H_{\mathcal{B}, L^{m}}\left(G^{m}\right)
$$

by which we may identify (up to isomorphism) both the topological and bornological Hochschild complexes with the complex $\left(\left\{H_{\mathcal{B}, L^{n+1}}\left(G^{n+1}\right)\right\}_{n \geq 0}, b_{*}\right)$, where $b_{n}$ is the unique additive extension of the Hochschild boundary map defined on elements $\left(g_{0}, g_{1}, \ldots, g_{n}\right)$ by the usual formula (1.1.1). With this isomorphism established for the Hochschild complex, the isomorphism for the cyclic and periodic cyclic complexes follows. It also dualizes to prove the corresponding result in cohomology.

In the above situation there is a third, simpler description of the cochain complex. Again, consider $H_{\mathscr{B}, L}(G)$. By Lemma 1.2.4, there is an isomorphism $H_{\mathscr{B}, L}(G)^{*} \cong$ $\operatorname{Hom}_{\mathscr{B}}(G, \mathbb{C})$. Passing to completed tensor powers and taking continuous duals results in an identification of both $C_{t}^{*}\left(H_{\mathcal{B}, L}(G)\right)$ and $C_{b}^{*}\left(H_{\mathcal{B}, L}(G)\right)$ with the cocomplex

$$
\begin{aligned}
C_{\mathcal{B}}^{*}(\mathbb{C}[G]):=\left\{f \in C^{n}(\mathbb{C}[G]) \mid \text { there exists } F \in \mathcal{B}\right. \text { with } \\
\left.\qquad\left|f\left(g_{0}, g_{1}, \ldots, g_{n}\right)\right| \leq F\left(\sum_{i=0}^{n} L\left(g_{i}\right)\right)\right\}_{n \geq 0} .
\end{aligned}
$$

The condition above on the right is simply the statement that $f$ is $\mathcal{B}$-bounded (with respect to the norm on $\mathbb{C}$ ). Thus this cocomplex is the subcocomplex of $C^{*}(\mathbb{C}[G])$ consisting of $\mathscr{B}$-bounded cochains; we denote its cohomology by $\mathrm{HH}_{\mathscr{B}}^{*}(\mathbb{C}[G])$. We note that the $B_{*}$ map of Section 1.1 is $\mathcal{B}$-bounded for all $\mathscr{B}$, so one can define the $\mathcal{B}$ cyclic cocomplex as

$$
\mathrm{CC}_{\mathscr{B}}^{*}(\mathbb{C}[G]):=\left\{f \in \mathrm{CC}^{n}(\mathbb{C}[G]) \mid f \text { is } \mathcal{B} \text {-bounded }\right\}_{n \geq 0}
$$

with corresponding cohomology groups denoted $\mathrm{HC}_{\mathscr{B}}^{*}(\mathbb{C}[G])$. And similarly with periodic cyclic cohomology. For any inclusion $\mathscr{B} \hookrightarrow \mathscr{B}^{\prime}$ of bounding sets there is an evident transformation of cohomology theories $F_{\mathscr{B}}^{*}(\mathbb{C}[G]) \rightarrow F_{\mathscr{B}^{\prime}}^{*}(\mathbb{C}[G])$ which preserves all of the standard structure maps relating the different groups; in particular, it induces a map of Connes-Gysin sequences. To further understand these cohomology groups, we introduce some additional terminology.

By a $\mathcal{B}$-simplicial set we mean a simplicial set $X$. together with weight functions $w_{n}: X_{n} \rightarrow \mathbb{R}_{+}$such that the face and degeneracy maps are $\mathcal{B}$-bounded. As before, let $(G, L)$ be a discrete group $G$ equipped with a proper word-length function $L$. A $\mathscr{B}-G$-set consists of a weighted set $\left(S, w_{S}\right)$ together with a specified left (resp. right) 
action of $G$ on $S$ such that there exists an $h \in \mathcal{B}$ with $w_{S}(g s)$ (resp. $w_{S}(s g)$ ) $\leq w_{S}(s)+h(L(g))$ for all $s \in S$ and $g \in G$ (in other words, the action is uniformly $\mathscr{B}$-bounded). A $\mathcal{B}$ - $G$-map between two $\mathcal{B}$-(left resp. right) $G$-sets is a $\mathscr{B}$-bounded map which is $G$-equivariant; it is a $\mathscr{B}-G$-isomorphism if it admits a two-sided inverse which is also a $\mathscr{B}-G$-map. Finally, a $\mathscr{B}-G$-bi-set is a weighted set equipped with both a left and right action of $G$ which is $\mathscr{B}$-bounded in the above sense, an isomorphism of two such having the obvious properties. The following are two basic examples relevant to this paper.

Example 1.3.5 (i) (The cyclic bar construction with coefficients). Let $(G, L)$ be as above and let $\left(S, w_{S}\right)$ be a $\mathcal{B}$ - $G$-bi-set. Then $N_{\bullet}^{\text {cy }}(G, S)=\left\{n \mapsto S \times G^{n}\right\}_{n \geq 0}$ with

$$
\begin{aligned}
\partial_{0}\left(s, g_{1}, \ldots, g_{n}\right) & =\left(s g_{1}, g_{2}, \ldots, g_{n}\right), \\
\partial_{i}\left(s, g_{1}, \ldots, g_{n}\right) & =\left(s, g_{1}, \ldots, g_{i} g_{i+1}, \ldots, g_{n}\right), \quad 1 \leq i \leq n-1, \\
\partial_{n}\left(s, g_{1}, \ldots, g_{n}\right) & =\left(g_{n} s, g_{1}, g_{2}, \ldots, g_{n-1}\right), \\
s_{j}\left(s, g_{1}, \ldots, g_{n}\right) & =\left(s, \ldots, g_{j}, 1, g_{j+1}, \ldots, g_{n}\right) .
\end{aligned}
$$

The simplicial weight is given by $w_{n}\left(s, g_{0}, \ldots, g_{n}\right)=w_{S}(s)+\sum_{i=1}^{n} L\left(g_{i}\right)$. The condition on $\left(S, w_{S}\right)$ implies that $N_{\bullet}^{\text {cy }}(G, S)$ is a $\mathcal{B}$-simplicial set. When $\left(S, w_{S}\right)=$ $(G, L)$, we denote this $\mathscr{B}$-simplicial set simply by $N_{\bullet}^{\text {cy }}(G)$

Example 1.3.5 (ii) (The bar resolution with coefficients). Let $\left(S, w_{S}\right)$ be a right $\mathcal{B}-G$ set. Recall that the non-homogeneous bar resolution of $G$ is $E G_{\bullet}=\left\{n \mapsto G^{n+1}\right\}_{n \geq 0}$ with

$$
\begin{aligned}
\partial_{i}\left[g_{0}, \ldots, g_{n}\right] & =\left[g_{0}, \ldots, g_{i} g_{i+1}, \ldots, g_{n}\right], \quad 0 \leq i \leq n-1, \\
\partial_{n}\left[g_{0}, \ldots, g_{n}\right] & =\left[g_{0}, \ldots, g_{n-1}\right], \\
s_{j}\left[g_{0}, \ldots, g_{n}\right] & =\left[g_{0}, \ldots, g_{j}, 1, g_{j+1}, \ldots, g_{n}\right] .
\end{aligned}
$$

The simplicial weight function on $E G$. is given by $w\left(\left[g_{0}, \ldots, g_{n}\right]\right)=\sum_{i=0}^{n} L\left(g_{i}\right)$. The left $G$-action is given, as usual, by $g\left[g_{0}, g_{1}, \ldots, g_{n}\right]=\left[g g_{0}, g_{1}, \ldots, g_{n}\right]$. Note that with respect to the given weight function and action of $G, E G$. is a $\mathcal{B}$-simplicial $G$-set for any $\mathcal{B}$. Viewing $S$ as a simplicial set with trivial simplicial structure, we can form the simplicial set $S \times E G$. with diagonal simplicial structure. Then $S \times{ }_{G} E G$. is the quotient of $S \times E G$. by the relation $\left(s g,\left[g_{0}, \ldots, g_{n}\right]\right) \simeq\left(s,\left[g g_{0}, \ldots, g_{n}\right]\right)$. This is a $\mathscr{B}$-simplicial set where the simplicial weight is given by

$$
w\left(\left[s,\left[g_{0}, \ldots, g_{n}\right]\right]\right):=\inf _{g \in G}\left(w_{S}\left(s g^{-1}\right)+L\left(g g_{0}\right)+\sum_{i=1}^{n} L\left(g_{i}\right)\right) .
$$

To a given $\mathcal{B}$-simplicial set $\left(X_{\bullet}, w_{\bullet}\right)$ one can associate the cochain cocomplex $C_{\mathscr{B}}^{*}(X, \mathbb{C})$ of $\mathcal{B}$-bounded singular cochains, where

$C_{\mathscr{B}, w .}^{n}(X ., \mathbb{C})=\left\{f: X_{n} \rightarrow \mathbb{C} \mid f\right.$ is $\mathcal{B}$-bounded with respect to the norm on $\left.\mathbb{C}\right\}$. 
This is naturally a subcocomplex of the usual cocomplex of singular cochains on $X$. with coefficients in $\mathbb{C}$. The $B$-cohomology of $X$. (with respect to the weight function $\left.w_{\bullet}\right)$ is $H_{\mathcal{B}, w_{\bullet}}^{*}\left(X_{\bullet}\right):=H^{*}\left(C_{\mathscr{B}, w_{\bullet}}^{*}\left(X_{\bullet}, \mathbb{C}\right)\right)$. The following is now evident.

Lemma 1.3.6. Under the natural isomorphism $C^{*}(\mathbb{C}[G]) \cong C^{*}\left(N_{\bullet}^{\text {cy }}(G), \mathbb{C}\right)$, we have a natural identification

$$
C_{t}^{*}\left(H_{\mathcal{B}, L}(G)\right) \cong C_{b}^{*}\left(H_{\mathcal{B}, L}(G)\right) \cong C_{\mathcal{B}, w_{\bullet}}^{*}\left(N_{\bullet}^{\mathrm{cy}}(G), \mathbb{C}\right)
$$

for all $(G, L)$ and countable $\mathcal{B}$.

For convenience we denote the groups $C_{\mathcal{B}, w}^{*}\left(N_{\bullet}^{\text {cy }}(G), \mathbb{C}\right)$ simply by $C_{\mathcal{B}}^{*}\left(N_{\bullet}^{\text {cy }}(G)\right)$.

1.4. The decomposition. As in the case of the group algebra, the Hochschild, cyclic and periodic cyclic (co)homologies of the afore-mentioned rapid decay algebras admit a decomposition indexed on $\langle G\rangle$, the set of conjugacy classes of $G$. The starting point is the following simple

Proposition 1.4.1. For any class of bounding functions $\mathscr{B}$, there is an isomorphism of $\mathscr{B}$-simplicial sets

$$
N_{\cdot}^{\text {cy }}(G) \cong \coprod_{x \in\langle G\rangle}\left(N_{\cdot}^{\text {cy }}(G)_{x}\right) \cong \coprod_{x \in\langle G\rangle} S_{x} \times_{G} E G .
$$

(cf. 1.3.5 (i), (ii) above), where ( $\left.N_{\bullet}^{\text {cy }}(G)_{x}\right)$ denotes the $\mathcal{B}$-subsimplicial set of $N_{\bullet}^{\text {cy }}(G)$ with $\left(N_{.}^{\text {cy }}(G)_{x}\right)_{n}=\left\{\left(g_{0}, \ldots, g_{n}\right) \mid\left\langle\prod_{i=0}^{n} g_{i}\right\rangle=x\right\}$, and $S_{x}=\{g \in G \mid\langle g\rangle=x\}$ with weight function induced by $L$ and the inclusion into $G$. The right $G$-action on $S_{x}$ is given by $h(g):=g^{-1} h g$ (conjugation by $g^{-1}$ ).

Proof. This equivalence is well-known, and the first decomposition is clearly a $\mathscr{B}$ simplicial isomorphism. It remains to note that the maps

$$
\begin{gathered}
\left(N_{\bullet}^{\mathrm{cy}}(G)_{x}\right)_{n} \ni\left(g_{0}, g_{1}, \ldots, g_{n}\right) \mapsto\left[\left(\prod_{i=0}^{n} g_{i}\right),\left[g_{0}, \ldots, g_{n}\right]\right] \\
=\left[\left(\prod_{i=0}^{n} g_{i}\right)^{g_{0}^{-1}},\left[1, \ldots, g_{n}\right]\right] \in S_{x} \times_{G} E G_{n} \\
S_{x} \times_{G} E G_{n} \ni\left[g,\left[1, \ldots, g_{n}\right]\right] \mapsto\left(\left(\prod_{i=1}^{n} g_{i}\right)^{-1} g, g_{1}, \ldots, g_{n}\right)
\end{gathered}
$$

are $\mathscr{B}$-bounded for any $\mathscr{B}$.

Together with the results of the previous section, we have the following immediate consequence. 
Corollary 1.4.2. For any conjugacy class $x$ the decomposition of (1.4.1) induces factorizations in homology and cohomology

$$
\begin{aligned}
\bigoplus_{x \in\langle G\rangle} F_{*}^{t}\left(H_{\mathcal{B}, L}(G)\right)_{x} & \rightarrow F_{*}^{t}\left(H_{\mathcal{B}, L}(G)\right) \rightarrow \prod_{x \in\langle G\rangle} F_{*}^{t}\left(H_{\mathcal{B}, L}(G)\right)_{x}, \\
\bigoplus_{x \in\langle G\rangle} F_{t}^{*}\left(H_{\mathcal{B}, L}(G)\right)_{x} & \longrightarrow F_{t}^{*}\left(H_{\mathcal{B}, L}(G)\right) \rightarrow \prod_{x \in\langle G\rangle} F_{t}^{*}\left(H_{\mathcal{B}, L}(G)\right)_{x}
\end{aligned}
$$

for $F=\mathrm{HH}, \mathrm{HC}, \mathrm{HPer}$, and with the maps in the Connes-Gysin sequence preserving this decomposition. These summands are preserved by homomorphisms coming from an inclusion of algebras $H_{\mathcal{B}, L}(G) \hookrightarrow H_{\mathcal{B}^{\prime}, L}(G)$ (induced by the relation $\mathcal{B}^{\prime} \prec \mathscr{B}$ ). Moreover there exists, for each $x$, isomorphisms

$$
\mathrm{HH}_{t}^{*}\left(H_{\mathcal{B}, L}(G)\right)_{x} \cong H_{\mathcal{B}}^{*}\left(S_{x} \times_{G} E G_{.}\right) .
$$

We note that the homomorphism $F_{*}^{t}\left(H_{\mathcal{B}, L}(G)\right) \rightarrow \prod_{x \in\langle G\rangle} F_{*}^{t}\left(H_{\mathcal{B}, L}(G)\right)_{x}$ may not be injective (with the same remark holding for the cohomology groups). In this paper, however, we will only be concerned with the summands indicated in the sums on the left.

Fix a conjugacy class $x$ and group element $h$ with $\langle h\rangle=x$, let $G_{h}$ denote the centralizer of $h$ in $G$, and $G_{h} \backslash G$ the corresponding right coset space. This is a weighted set, with weight $\bar{L}\left(G_{h} g\right):=\min _{g^{\prime} \in G_{h}} L\left(g^{\prime} g\right)$. The right $G$-action on $G_{h} \backslash G$ is the obvious one.

Proposition 1.4.3. The natural map $\pi_{x}: G_{h} \backslash G \rightarrow S_{x}$ given by $G_{h} g \mapsto g^{-1} h g$ is a $G$-equivariant isomorphism of right $G$-sets and $\mathcal{B}$-bounded.

Proof. It is obviously a $G$-equivariant isomorphism. It is also linearly bounded in terms of the respective weight functions.

Unfortunately, and here is the key point, this map is not a $\mathcal{B}-G$-isomorphism of right $\mathcal{B}-G$-sets in general. In order to proceed beyond Corollary 1.4 .2 and get an identification analogous to Theorem 1.1.2 (ii), we will need to impose conditions on $(G, L)$. We say that $(G, L)$ has a $\mathcal{B}$-solvable conjugacy bound at the conjugacy class $x=\langle h\rangle \in\langle G\rangle$ if there exists $f \in \mathscr{B}$ with the property: for each $y \in S_{x}$, there exists $g=g_{y} \in G$ with $y=h^{g_{y}}$ and $L\left(g_{y}\right) \leq f(L(y))$. In other words, there exists a map $\psi_{x}: S_{x} \ni y \mapsto\left[g_{y}\right] \in G_{h} \backslash G$ which is an inverse to $\pi_{x}$ and is $\mathcal{B}$-bounded. As $\pi_{x}$ is $G$-equivariant, so is $\psi_{x}$. The pair $(G, L)$ has a $\mathcal{B}$-solvable conjugacy bound if it does so at each non-elliptic conjugacy class $x$.

Lemma 1.4.4. The pair $(G, L)$ has a $\mathcal{B}$-solvable conjugacy bound at $x$ iff $\pi_{x}$ is a $\mathscr{B}-G$-isomorphism of right $\mathcal{B}-G$-sets. In this case, there is a natural isomorphism of $B$-simplicial sets

$$
\left(\pi_{x}\right)_{.}: G_{h} \backslash G \times_{G} E G . \underset{\mathscr{B}}{\cong} S_{x} \times_{G} E G_{\bullet},
$$

which induces an isomorphism on $\mathfrak{B}$-bounded cohomology. 
Proof. Given the definitions of the respective simplicial weight functions, it suffices to check that $\left(\pi_{x}\right)_{1}$ is a $\mathscr{B}$-isomorphism, which is implied by the fact $\pi_{x}$ is a $\mathscr{B}$ isomorphism.

Proposition 1.4.5. For each $h$ the inclusion $G_{h} \hookrightarrow G$ yields an inclusion of $\mathscr{B}$ simplicial sets

$$
B G_{h}=\left(G_{h} \backslash G\right) \times_{G_{h}} E\left(G_{h}\right) . \hookrightarrow G_{h} \backslash G \times_{G} E G .
$$

which induces an isomorphism on B-bounded cohomology groups.

Proof. This result is shown in [Og2] for $\mathscr{B}=\mathcal{P}$, but the same argument works in this more general case (this point is also noted in [Me1] for $\mathcal{P}$ and $\mathcal{E}$ ).

Combining 1.4.2 and 1.4.5 we have

Corollary 1.4.6. If the pair $(G, L)$ has a $B$-solvable conjugacy bound, then for each non-elliptic conjugacy class $x=\langle h\rangle$, there is an isomorphism of cohomology groups

$$
\mathrm{HH}_{t}^{*}\left(H_{\mathcal{B}, L}(G)\right)_{x} \cong H_{\mathcal{B}}^{*}\left(S_{x} \times_{G} E G .\right) \cong H_{\mathcal{B}}^{*}\left(B G_{h} ; \mathbb{C}\right) .
$$

We observe that having a $\mathscr{B}$-solvable conjugacy bound is a local condition, and strictly weaker than requiring that the $G$-map

$$
\bigsqcup_{x=\langle h\rangle \in\langle G\rangle}\left(G_{h} \backslash G\right) \stackrel{\amalg_{x=\langle h\rangle \in\langle G\rangle} \pi_{x}}{\longrightarrow} \bigsqcup_{x=\langle h\rangle \in\langle G\rangle}\left(S_{x}\right)
$$

is a $\mathscr{B}$ - $G$-isomorphism. Given $G$, it is also possible that certain conjugacy classes have a $\mathscr{B}$-solvable conjugacy bound, while others are not. To illustrate, $\langle 1\rangle$ has a $\mathscr{B}$-solvable conjugacy bound for any $G$ and bounding class $\mathscr{B}$, for trivial reasons. This statement is false in general for conjugacy classes $x \neq\langle 1\rangle$.

Borrowing some terminology from $[\mathrm{Me} 2]$, we say that $(G, L)$ is $\mathscr{B}$-isocohomological if the transformation $H_{\mathcal{B}}^{*}(B G ; \mathbb{C}) \rightarrow H^{*}(B G ; \mathbb{C})$ (induced by the inclusion $\left.\mathscr{B} \hookrightarrow \mathcal{B}_{\max }\right)$ is an isomorphism. Altogether we have the following result.

Theorem 1.4.7. Let $(G, L)$ be a discrete group equipped with proper word-length function L. Suppose that there exists a countable bounding class $\mathbb{B}$ such that

(i) each non-elliptic conjugacy class $x$ has a $B$-solvable conjugacy bound, and

(ii) $G_{h}$ is $\mathscr{B}$-isocohomological for each non-elliptic $x=\langle h\rangle \in\langle G\rangle$.

Then for each such $x=\langle h\rangle \in\langle G\rangle$ there are isomorphisms

$$
\begin{aligned}
\mathrm{HH}_{t}^{*}\left(H_{\mathcal{B}, L}(G)\right)_{x} & =\mathrm{HH}_{\mathcal{B}}^{*}(\mathbb{C}[G])_{x} \cong \mathrm{HH}^{*}(\mathbb{C}[G])_{x}=H^{*}\left(B G_{h} ; \mathbb{C}\right), \\
\operatorname{HC}_{t}^{*}\left(H_{\mathcal{B}, L}(G)\right)_{x} & =\mathrm{HC}_{\mathcal{B}}^{*}(\mathbb{C}[G])_{x} \cong \mathrm{HC}^{*}(\mathbb{C}[G])_{x} .
\end{aligned}
$$


Proof. As we have seen, $\mathrm{HH}_{\mathcal{B}}^{*}(\mathbb{C}[G])_{x} \cong H_{\mathcal{B}}^{*}\left(B G_{h} ; \mathbb{C}\right)$ when $x$ has a $\mathcal{B}$-solvable conjugacy bound. Condition (ii) then implies that $H_{\mathcal{B}}^{*}\left(B G_{h} ; \mathbb{C}\right) \cong H^{*}\left(B G_{h} ; \mathbb{C}\right)$. The statement for cyclic cohomology follows by a comparison of Connes-Gysin sequences.

As with non-bounded Hochschild and cyclic cohomology groups, it is relatively easy to show that when $x$ has a $\mathscr{B}$-solvable conjugacy bound and is elliptic, there is an isomorphism

$$
\mathrm{HC}_{\mathscr{B}}^{*}(\mathbb{C}[G])_{x} \cong H_{\mathcal{B}}^{*}\left(B G_{h} ; \mathbb{C}\right) \otimes \mathrm{HC}^{*}(\mathbb{C}),
$$

where the $S^{*}$-map on the right is given by $\operatorname{Id} \otimes S_{\mathbb{C}}^{*}$. For $x$ non-elliptic, the situation is more delicate. The next lemma follows by a straightforward adoption of the method of Nistor [Ni1] (cf. [Ji2]). Since it is not needed for the rest of the paper, we state it without proof.

Lemma 1.4.8. Given $(G, L)$ satisfying the conditions of Theorem 1.4.7, and let $x=$ $\langle h\rangle \in\langle G\rangle$ be a non-elliptic class. Let $C_{h} \subset G_{h}$ denote the infinite cyclic subgroup generated by $h$, and let $L_{h}=\left.L\right|_{C_{h}}$. Let $\mathbb{Z}$ denote the infinite (multiplicative) cyclic group generated by $t$, with $L_{\mathbb{Z}}\left(t^{n}\right)=n$. If the isomorphism $\left(\mathbb{Z}, L_{\mathbb{Z}}\right) \rightarrow\left(C_{h}, L_{h}\right)$, $t \mapsto h$, is a B-isomorphism, then $\operatorname{HC}_{t}^{*}\left(H_{\mathcal{B}, L}(G)\right)_{x} \cong H_{\mathcal{B}}^{*}\left(N_{h} ; \mathbb{C}\right)$, where $N_{h}=$ $G_{h} / C_{h}$ equipped with the quotient word-length function coming from $G_{h}$.

Our main application of Theorem 1.4.7 is

Corollary 1.4.9. Suppose that $G$ is in the class $\mathcal{C}(\mathbb{C})$ and that $(G, L)$ satisfies the conditions of Theorem 1.4.7. Then $(G, L)$ is in the class $\mathcal{C}_{\mathcal{B}}(\mathbb{C})$. Consequently the conjecture $B$-SrBC holds for $G$.

In the next section we exhibit certain classes of groups to which this corollary applies.

\section{Geometric groups satisfying $\mathscr{B}$-SrBC}

2.1. Nilpotent and hyperbolic groups. In previous work, the first author has shown $(G, L)$ satisfies the $\mathcal{P}$-nilpotency condition for any proper word-length function $L$ on $G$ when $G$ is of polynomial growth [Ji1] or word-hyperbolic [Ji3]. We show that for word hyperbolic groups or finitely generated torsion free two-step nilpotent groups, these results can be efficiently recovered (and extended) using the machinery of Section 1.

Proposition 2.1.1. Let $G$ be a finitely generated torsion free two-step nilpotent group, and $L$ a proper word-length function on $G$. Then, for all bounding families $\mathscr{B} \supseteq \mathcal{P}$, 
(i) each conjugacy class $x$ has a $\mathcal{B}$-solvable conjugacy bound,

(ii) the centralizer $G_{h}$ for $h \in x$ is $B$-isocohomogical for each conjugacy class $x$, and

(iii) $G_{x} /(h)$ has finite rational cohomological dimension $\left(\operatorname{rcd}\left(G_{x} /(h)\right)<\infty\right)$ for each non-elliptic $x=\langle h\rangle$.

Hence $G$ satisfies $\mathcal{B}$-nilpotency for all $\mathfrak{B} \supseteq \mathcal{P}$.

Proof. For (i), we note that polynomial solvability of the conjugacy problem implies the existence of a $\mathcal{B}$-solvable conjugacy bound for all $\mathscr{B} \supseteq \mathcal{P}$. Note that by [Bl] the conjugacy problem for a finitely generated nilpotent group is solvable. We must then show that there is a polynomial solution. Consider the torsion-free nilpotent group $G$ given by the central extension $C \rightarrow G \rightarrow N$, with $C$ and $N$ free abelian groups, with bases $\left\{e_{1}^{\prime}, \ldots, e_{n}\right\}$ and $\left\{f_{1}^{\prime}, \ldots, f_{m}^{\prime}\right\}$, respectively. Let $g$ and $h$ be conjugate elements in $G$, with $u g=h u$. We can write these elements as $g=f_{1}^{x_{1}} f_{2}^{x_{2}} \ldots f_{m}^{x_{m}} c$, $h=f_{1}^{y_{1}} f_{2}^{y_{2}} \ldots f_{m}^{y_{m}} c^{\prime}$, and $u=f_{1}^{z_{1}} f_{2}^{z_{2}} \ldots f_{m}^{z_{m}}$ with $x_{i}, y_{i}$, and $z_{i}$ integers, $c$ and $c^{\prime}$ elements of $C$. We can also write $c=e_{1}^{a_{1}} \ldots e_{n}^{a_{n}}$, and $c^{\prime}=e_{1}^{b_{1}} \ldots e_{n}^{b_{n}}$, for integers $a_{i}$ and $b_{j}$. (Here we have identified $C$ with its image in $G$, and $f_{i}$ is the image of $f_{i}^{\prime}$ in $G$ under a fixed cross-section $N \rightarrow G$.) Now $f_{i}^{\prime} f_{j}^{\prime}=f_{j}^{\prime} f_{i}^{\prime}$ so that $f_{i} f_{j}=f_{j} f_{i} C_{i, j}$ for some $C_{i, j} \in C$. From $u g=h u$ we have $f_{1}^{z_{1}} f_{2}^{z_{2}} \ldots f_{m}^{z_{m}} f_{1}^{x_{1}} f_{2}^{x_{2}} \ldots f_{m}^{x_{m}} c=$ $f_{1}^{y_{1}} f_{2}^{y_{2}} \ldots f_{m}^{y_{m}} f_{1}^{z_{1}} f_{2}^{z_{2}} \ldots f_{m}^{z_{m}} c^{\prime}$. Applying the conjugation properties we obtain

$$
\begin{aligned}
f_{1}^{x_{1}+z_{1}} & f_{2}^{x_{2}+z_{2}} \ldots f_{m}^{x_{m}+z_{m}} C_{m, 1}^{x_{m} z_{1}} \ldots C_{1,1}^{x_{2} z_{1}} C_{m, 2}^{x_{m} z_{2}} \ldots C_{m, m-1}^{x_{m} z_{m-1}} c \\
& =f_{1}^{z_{1}+y_{1}} f_{2}^{z_{2}+y_{2}} \ldots f_{m}^{z_{m}+y_{m}} C_{m, 1}^{z_{m} y_{1}} \ldots C_{1,1}^{z_{2} y_{1}} C_{m, 2}^{z_{m} y_{2}} \ldots C_{m, m-1}^{z_{m} y_{m-1}} c^{\prime} .
\end{aligned}
$$

Moving all of the $f_{i}$ 's to one side we obtain $f_{1}^{x_{1}-y_{1}} f_{2}^{x_{2}-y_{2}} \ldots f_{m}^{x_{m}-y_{m}} C_{m, 1}^{x_{m} z_{1}-y_{1} z_{m}}$ $\ldots C_{1,1}^{x_{2} z_{1}-y_{1} z_{2}} C_{m, 2}^{x_{m} z_{2}-y_{2} z_{m}} \ldots C_{m, m-1}^{x_{m} z_{m-1}-y_{m-1} z_{m}} c c^{\prime-1}=e$. As $\left\{f_{1}^{\prime}, \ldots, f_{m}^{\prime}\right\}$ are free abelian generators for $N$, we see that $x_{i}=y_{i}$ for all $i$. The last equation reduces to $C_{m, 1}^{x_{m} z_{1}-x_{1} z_{m}} \ldots C_{1,1}^{x_{2} z_{1}-x_{1} z_{2}} C_{m, 2}^{x_{m} z_{2}-x_{2} z_{m}} \ldots C_{m, m-1}^{x_{m} z_{m-1}-x_{m-1} z_{m}} c c^{\prime-1}=e$. From this we obtain a linear system to determine the $z_{i}$ in terms of the $x_{j} \mathrm{~s}, a_{j} \mathrm{~s}$, and $b_{j} \mathrm{~s}$. Since $g$ and $h$ are conjugate, this system has a solution. Thus there is a solution which is bounded by a polynomial of the absolute values of the entries in the coefficient matrix of this system.

As $G$ is nilpotent, so is $G_{x}$ for all $x$. Choose an element $z \in C$ with ord $(z)=\infty$. Let $C_{z}$ denote the infinite cyclic subgroup generated by $z$. Associated to the shortexact sequence of groups $\mathbb{Z}=C_{z} \succ G \rightarrow \bar{G}$ is a Serre spectral sequence in $\mathscr{B}$-cohomology (the case $\mathscr{B}=\mathcal{P}$ is done in detail in [Og2], the more general case follows by similar reasoning, with similar restrictions, cf. [Og3]). By a spectral sequence comparison together with induction on $n$, we see that nilpotent groups are $\mathscr{B}$-isocohomological for all $\mathscr{B} \supseteq \mathcal{P}$. Finally, any finitely generated nilpotent group $G^{\prime}$ satisfies $\operatorname{rcd}\left(G^{\prime}\right)<\infty$; as subquotients of finitely generated nilpotent groups are again finitely generated nilpotent, this implies (iii). 
Proposition 2.1.2. Let $G$ be a word-hyperbolic group and $L$ a proper word-length function on $G$. Then, for all bounding families $\mathfrak{B} \supseteq \mathscr{L}$,

(i) each conjugacy class $x$ has a $\mathscr{B}$-solvable conjugacy bound,

(ii) the centralizer $G_{h}$ is $\mathcal{B}$-isocohomogical for each element $h$ in the non-elliptic conjugacy class $x$, and

(iii) $\operatorname{rcd}\left(G_{h} /(h)\right)<\infty$ for each non-elliptic class $x=\langle h\rangle$.

Hence $G$ satisfies $\mathcal{B}$-nilpotency for all $\mathfrak{B} \supseteq \mathscr{L}$.

Proof. In the case of word-hyperbolic groups, the situation is even nicer. If $x$ is a non-elliptic conjugacy class, then the conjugacy bound in this case is linear [Gr], $[\mathrm{Bu}]$. Moreover, $G_{h}$ is virtually cyclic and isometrically embedded in $G$ for any $h \in x$, implying that it is $\mathcal{B}$-isocohomological [Ji1], [Og2]. It also implies that the quotient $G_{h} /(h)$ is finite, so $\operatorname{rcd}\left(G_{h} /(h)\right)=0$.

2.2. Relatively hyperbolic groups. We begin by recalling the basic setup for relatively hyperbolic groups. Thus, let $G$ be a discrete group and $\left\{H_{\lambda}\right\}_{\lambda \in \Lambda}$ a family of subgroups of $G$. Set $\mathscr{H}=\bigcup_{\lambda \in \Lambda} H_{\lambda} \backslash\left\{1_{G}\right\}$. A subset $X \subset G$ is a relative generating set of $G$, with respect to $\left\{H_{\lambda}\right\}_{\lambda \in \Lambda}$, if $G=\langle X \cup \mathcal{H}\rangle$. In this case, $G$ can be considered as the quotient of a free product $F=F(X) *\left(*_{\lambda \in \Lambda} H_{\lambda}\right)$, where $F(X)$ is the free group with basis $X$.

Let $\mathcal{R} \subset F$ be such that the normal closure of $\mathcal{R}$ is the kernel of the quotient map. In this case $G$ has a relative presentation with respect to $\left\{H_{\lambda}\right\} \lambda \in \Lambda$ given by $\langle X, \mathscr{H} \mid R=1, R \in \mathcal{R}\rangle$. The group $G$ is finitely relatively presented if $\mathcal{R}$ is finite.

Let $W$ be a path in $F$ representing $1_{G} \in G$. Then $W$ can be represented in the following form: $W=\prod_{i=1}^{k} f_{i} R_{i}^{ \pm 1} f_{i}^{-1}$, where the $f_{i} \in F$ and $R_{i} \in \mathcal{R}$. We can then define the relative area of such a path:

$$
\begin{array}{r}
\operatorname{area}^{\mathrm{rel}}(W)=\min \left\{k \in \mathcal{N} \mid W \text { can be written as } \prod_{i=1}^{k} f_{i} R_{i}^{ \pm 1} f_{i}^{-1}\right. \\
\text { where } \left.f_{i} \in F \text { and } R_{i} \in \mathcal{R}\right\} .
\end{array}
$$

The group $G$ is relatively hyperbolic with respect to $\left\{H_{\lambda}\right\}_{\lambda \in \Lambda}$ if $G$ is finitely relatively presented with respect to $\left\{H_{\lambda}\right\}_{\lambda \in \Lambda}$ and there is a constant $C$ such that for any word $W \in F$ representing $1_{G} \in G$ we have that area ${ }^{\text {rel }}(W) \leq C\|W\|$, where $\|W\|$ is the length of $W$ in $F$ [Os]. In particular if $G$ is relatively hyperbolic with respect to $\left\{H_{\lambda}\right\} \lambda \in \Lambda$, then the Cayley graph $\Gamma(G, X \cup \mathscr{H})$ is a hyperbolic metric space, in the graph metric.

An alternate description is given by Farb in [Fa]. Let $G$ be a finitely generated group, and let $\left\{H_{\lambda}\right\}_{\lambda \in \Lambda}$ be a family of finitely generated subgroups of $G$. Fix $X$, a finite symmetric set of generators of $G$. To the corresponding Cayley graph $\Gamma(G, X)$ add a vertex $v\left(g H_{\lambda}\right)$ for each left coset of each $H_{\lambda}$ in the family, and connect $v\left(g H_{\lambda}\right)$ with the vertex corresponding to each element $x \in g H_{\lambda}$ by an edge of length $1 / 2$. The resulting graph $\widehat{\Gamma}\left(G, X ;\left\{H_{\lambda}\right\} \lambda \in \Lambda\right)$ is called the coned-off Cayley graph of $G$ 
relative to $\left\{H_{\lambda}\right\}_{\lambda \in \Lambda}$. In Farb's notation, the group $G$ is weakly relative hyperbolic with respect to $\left\{H_{\lambda}\right\}_{\lambda \in \Lambda}$ if $\widehat{\Gamma}\left(G, X ;\left\{H_{\lambda}\right\}_{\lambda \in \Lambda}\right)$ is a hyperbolic metric space. As $\hat{\Gamma}\left(G, X ;\left\{H_{\lambda}\right\}_{\lambda \in \Lambda}\right)$ is quasi-isometric to $\Gamma(G, X \cup \mathcal{H})$, this property is weaker than that defined above. However, when augmented with the bounded coset penetration property (defined below), this notion is equivalent to relative hyperbolicity.

Definition. Let a group $G$ be weakly relatively hyperbolic to a family $\left\{H_{\lambda}\right\}_{\lambda \in \Lambda}$ of finitely generated subgroups. $\left(G,\left\{H_{\lambda}\right\}_{\lambda \in \Lambda}\right)$ is said to satisfy the Bounded Coset Penetration (BCP) property if for each constant $k$ there exists a constant $c=c(k)$ such that for every pair of $k$-quasi-geodesics $p$ and $q$ in the coned-off Cayley graph with the same endpoints and without backtracking the following holds:

(i) If $p$ penetrates a coset $g H_{\lambda}$ and $q$ does not penetrate $g H_{\lambda}$, then the point at which $p$ enters the coset is at most a $\Gamma(G, X)$-distance of $c(k)$ from the point at which $p$ leaves the coset.

(ii) If $p$ and $q$ both penetrate a coset $g H_{\lambda}$, then the points at which $p$ and $q$ enter $g H_{\lambda}$ are at most a $\Gamma(G, X)$-distance of $c(k)$ from each other. Similarly the points at which $p$ and $q$ exit the coset are within a $\Gamma(G, X)$-distance of $c(k)$ from one-another.

Definition. Let $G$ be a finitely generated group which is relatively hyperbolic with respect to the collection of subgroups $\left\{H_{\lambda}\right\}_{\lambda \in \Lambda}$. An element of $G$ is parabolic if it is conjugate to an element in one of the $H_{\lambda}$. Otherwise the element is said to be hyperbolic.

Proposition 2.2.1. Let $G$ be a finitely generated group, $\left\{H_{\lambda}\right\}_{\lambda \in \Lambda}$ a collection of nontrivial subgroups of $G$. Suppose that $G$ is finitely presented with respect to $\left\{H_{\lambda}\right\}_{\lambda \in \Lambda}$. Then the collection $\left\{H_{\lambda}\right\}_{\lambda \in \Lambda}$ is finite. In particular, if $G$ is relatively hyperbolic with respect to a collection $\left\{H_{\lambda}\right\}_{\lambda \in \Lambda}$ of nontrivial subgroups, then card $\Lambda<\infty$.

Proof. This is Corollary 2.48 of [Os].

Proposition 2.2.2. Let $G$ be a finitely generated group with generating set $X$ such that $G$ is relatively hyperbolic with respect to $\left\{H_{\lambda}\right\}_{\lambda \in \Lambda}$, and let $h \in G$ be a nontorsion element which does not conjugate into any of the $H_{\lambda}$ (such an element is said to be hyperbolic). Then the centralizer $G_{h}$ is finitely generated, virtually cyclic, and the embedding $G_{h} \rightarrow G$ is a quasi-isometric embedding.

Proof. By Theorem 4.19 of [Os], $G_{h}$ is a strongly relatively quasi-convex subgroup of $G$. Theorem 4.13 of [Os] shows that $G_{h}$ is finitely generated and that the inclusion $G_{h} \rightarrow G$ is a quasi-isometric embedding. Theorem 4.16 of [Os] gives that $G_{h}$ is a word-hyperbolic group. As the infinite cyclic subgroup generated by $h$ lies in the center of the hyperbolic group $G_{h}$, we have that $G_{h}$ is virtually cyclic. 
Proposition 2.2.3. Let $G$ be a finitely generated group with generating set $X$ such that $G$ is relatively hyperbolic with respect to $\left\{H_{\lambda}\right\}_{\lambda \in \Lambda}$, and let $h \in G$ be a nontorsion parabolic element such that $h \in x H_{\lambda} x^{-1}$. Then $G_{h}=x H_{\lambda, x^{-1} h x^{x^{-1}}}$, where $H_{\lambda, x^{-1} h x}$ is the centralizer of $x^{-1} h x$ in $H_{\lambda}$.

Proof. This is due to the fact that $H_{\lambda}$ and $x H_{\lambda} x^{-1}$ are conjugated by $x$.

Proposition 2.2.4. Let $G$ be a finitely generated group with generating set $X$ such that $G$ is relatively hyperbolic with respect to $\left\{H_{\lambda}\right\}_{\lambda \in \Lambda}$, and let $\mathscr{H}=\bigcup_{\lambda \in \Lambda} H_{\lambda} \backslash\left\{1_{G}\right\}$. Then for every $k$ there is an $\varepsilon=\varepsilon(k)$ such that given any two $k$-quasi-geodesics without backtracking in $\Gamma(G, X \cup \mathcal{H})$, say $p$ and $q$, the following conditions hold:

(1) If p penetrates a coset $g H_{\lambda}$ and $q$ does not penetrate $g H_{\lambda}$, then the point at which $p$ enters the coset is at most a $\Gamma(G, X)$-distance of $\varepsilon(k)$ from the point at which $p$ leaves the coset.

(2) If $p$ and $q$ both penetrate a coset $g H_{\lambda}$, then the points at which $p$ and $q$ enter $g H_{\lambda}$ are at most a $\Gamma(G, X)$-distance of $\varepsilon(k)$ from each other. Similarly the points at which $p$ and $q$ exit the coset are within a $\Gamma(G, X)$-distance of $\varepsilon(k)$ from one-another.

Thus $\varepsilon$ works for the constant $c$ in the definition of the BCP property.

Proof. This is Theorem 3.23 of [Os].

Lemma 2.2.5. Let $(H, d)$ be a $\delta$-hyperbolic geodesic metric space. For every $k$ there is a constant $\tilde{N}=\tilde{N}(k)$ such that if $p$ is a $k$-quasigeodesic with endpoints $x$ and $y$, then $p$ lies within the $\tilde{N}$-neighborhood of every geodesic $[x, y]$ joining $x$ to $y$ in $H$. Moreover $\tilde{N}$ can be bounded by a polynomial in $k$.

Proof. This is Theorem 1.7 of Part III.H of [BH]. In [BH] actually only the first statement above is contained, but the existence of the polynomial follows from the proof. In particular,

$$
\begin{aligned}
\tilde{N}(k) \leq & \left(\delta \log _{2}\left(2 k^{3}+6 k^{2}+3 k+2\right)\right. \\
& \left.+\delta \log _{2}\left[\delta \log _{2}\left(2 k^{3}+6 k^{2}+3 k+2\right)\right]+1\right)\left(k^{2}+1\right)+\frac{1}{2}\left(2 k^{3}+3 k\right),
\end{aligned}
$$

which is clearly bounded by a polynomial in $k$.

Corollary 2.2.6. Let $(H, d)$ be a $\delta$-hyperbolic geodesic metric space. For every $k$ and $R$ there is a constant $N=N(k, R)$ such that if $p$ and $q$ are $k$-quasigeodesics whose starting points are within $R$ from each other, and whose ending points are within $R$ from each other, then $p$ and $q$ lie within the $N$-neighborhood of each other. Moreover $N(k, R)$ can be bounded by a polynomial in $k$ and $R$. 
Proof. Denote the starting and ending points of $p$ ( resp. $q$ ) by $x$ and $y$ (resp. $x^{\prime}$ and $\left.y^{\prime}\right)$. By Lemma 2.2.5, $p$ and $[x, y]$ lie within an $\widetilde{N}(k)$-neighborhood of each other. Similarly for $q$ and $\left[x^{\prime}, y^{\prime}\right]$. Consider the geodesic quadrilateral $x, y, y^{\prime}, x^{\prime}$. The sides $\left[x, x^{\prime}\right]$ and $\left[y, y^{\prime}\right]$ each have length less than $R$ by hypothesis. For any point on $[x, y]$ which is at least $R+2 \delta$ from either endpoint, hyperbolicity gives a point on $\left[x^{\prime}, y^{\prime}\right]$ which is within a distance of $2 \delta$. Similarly for points on $\left[x^{\prime}, y^{\prime}\right]$ at least $R+2 \delta$ from either endpoint. As every point of $[x, y]$ is within $R+2 \delta$ from such a point, it follows that $[x, y]$ and $\left[x^{\prime}, y^{\prime}\right]$ lie in $(R+4 \delta)$-neighborhoods of each other. It follows that $N(k, R)=\tilde{N}(k)+R+2 \delta$ works. Moreover, as $\tilde{N}(k)$ is bounded by a polynomial in $k$, we have that $N(k, R)$ is bounded by a polynomial in $k$ and $R$.

Proposition 2.2.7. Let $G, \mathcal{H}$ and $\varepsilon$ be as in Proposition 2.2.4. Then $\varepsilon(k)$ is bounded by a polynomial in $k$.

Proof. In [Os] the proof of Theorem 3.23 bounds $\varepsilon(k)$ by the maximum of three terms, each of which can be expressed as a polynomial in $N=N(k)$. By the above lemma, this is bounded by a polynomial in $k$. In particular, $\varepsilon(k)=\max \left\{\varepsilon^{\prime}(k), C^{\prime}(k), D(k)\right\}$, where $C^{\prime}(k)=L M\left(1+k\left(2 \varepsilon^{\prime}(k)+1\right)+2 \varepsilon^{\prime}(k)\right), D(k)=L M\left(1+k\left(2 \varepsilon^{\prime}(k)+\right.\right.$ $\left.1)+2 \varepsilon^{\prime}(k)+k+1\right), \varepsilon^{\prime}(k)=2 K(k)^{2} L M(4 k+1)$, for $L$ and $M$ positive constants depending only on the finite relative presentation $\langle X, \mathscr{H} \mid R=1, R \in \mathcal{R}\rangle$. Let $K_{0}=N(k, 0)$, where $N(k, R)$ is the constant from Corollary 2.2.6. Then $K(k)=$ $N\left(k, K_{0}\right)+\frac{1}{2}$ is the $K(k)$ showing up in the definition of $\varepsilon^{\prime}$. It is clear that $\varepsilon(k)$ is bounded by a polynomial in $k$.

The following is, at heart, the pseudo-Anosov case of Theorem 4.1 of [Ma].

Lemma 2.2.8. Let $u$ and $v$ be conjugate non-torsion hyperbolic elements of $G$. Then there exist a constant $K_{h}$ and $g \in G$ with $u=g^{-1} v g$ and $\ell_{\widehat{\Gamma}}(\hat{g}) \leq K_{h}\left(\ell_{\Gamma}(u)+\right.$ $\left.\ell_{\Gamma}(v)\right)$, where $\hat{g}$ is the "shortened path" in the relative graph $\hat{\Gamma}$ corresponding to the element $g \in G$. Moreover, $K_{h}$ is independent of the choice of $u$ and $v$.

Proof. By the work in [Os] on translation length of hyperbolic elements, there exists a $d>0$ such that for any hyperbolic element $h \in G$ one has $\ell_{\widehat{\Gamma}}\left(\widehat{h^{n}}\right) \geq|n| d$. Moreover, for any $x \in G$ we have $d_{\widehat{\Gamma}}\left(x, h^{n} x\right) \geq|n| d$.

Let $\alpha$ be a quasi-axis for the hyperbolic element $h$, acting on $\widehat{\Gamma}$ as a hyperbolic isometry. That is, $\alpha$ is a bi-infinite geodesic such that for every $n, h^{n} \alpha$ lies in a $2 \delta$ neighborhood of $\alpha$, where $\delta$ is the hyperbolicity constant of $\widehat{\Gamma}$.

If $x \in G$ then there exists constant $K$ such that $d_{\widehat{\Gamma}}(x, \alpha) \leq K d_{\widehat{\Gamma}}(x, h x)$, for $K$ independent of $x$ and $h$. This is Lemma 4.3 of [Ma], which relies only on $\widehat{\Gamma}$ being a hyperbolic metric space, on which $h$ acts as a hyperbolic isometry.

Thus if $u$ and $v$ are two conjugate hyperbolic elements of $G$, let $g$ be some conjugator with $u=g^{-1} v g$. Let $\alpha$ and $\beta$ be the axes of $u$ and $v$, respectively, as hyperbolic isometries. Let $a$ be the closest point on $\alpha$ to the identity element, 
$e$, and let $b$ be the closest point on $\beta$ to $e$. Applying our above inequality yields $\left.d_{\widehat{\Gamma}}(e, a) \leq K d_{(} e, u\right)=K \ell_{\widehat{\Gamma}}(\hat{u})$ and $d_{\widehat{\Gamma}}(e, b) \leq K \ell_{\hat{\Gamma}}(\hat{v})$. The conjugator $g$ takes the axis $\beta$ to the axis $\alpha$, so $g b \in \alpha$. The translation length of $u$ is at most $\ell_{\widehat{\Gamma}}(\hat{u})$, so there is an $n$ such that $d_{\widehat{\Gamma}}\left(a, u^{n} g b\right) \leq \ell_{\widehat{\Gamma}}(\hat{u})$, as an orbit of $u$ is $\ell_{\widehat{\Gamma}}(\hat{u})$-dense on $\alpha$. Let $g^{\prime}=u^{n} g$. Then $g^{\prime}$ serves to conjugate $u$ to $v$. Moreover, $d_{\widehat{\Gamma}}\left(e, g^{\prime}\right) \leq d_{\widehat{\Gamma}}(e, a)+$ $d_{\widehat{\Gamma}}\left(a, g^{\prime} b\right)+d_{\widehat{\Gamma}}\left(g^{\prime} b, g^{\prime}\right)$. Thus $\ell_{\widehat{\Gamma}}\left(\hat{g}^{\prime}\right)=d_{\widehat{\Gamma}}\left(e, g^{\prime}\right) \leq K \ell_{\widehat{\Gamma}}(\hat{u})+\ell_{\widehat{\Gamma}}(\hat{u})+K \ell_{\widehat{\Gamma}}(\hat{v})$. The lemma follows by observing that $\ell_{\Gamma}(u) \geq \ell_{\hat{\Gamma}}(\hat{u})$ and $\ell_{\Gamma}(v) \geq \ell_{\hat{\Gamma}}(\hat{v})$.

Lemma 2.2.9. Let $u$ be a non-torsion parabolic element of $G$ lying in $\gamma H \gamma^{-1}$. Then there exists a universal polynomial $K_{p}$ of degree one such that $\ell_{\hat{\Gamma}}(\hat{\gamma}) \leq K_{p}\left(\ell_{\Gamma}(u)\right)$.

Proof. For a coset $\gamma H$, let $\ell_{\Gamma}^{*}(\gamma H)=\min \left\{\ell_{\Gamma}(x h) \mid h \in H\right\}$. Let $x$ be a point of $\gamma H$ such that $\ell_{\Gamma}(x)=\ell_{\Gamma}^{*}(\gamma H)$. By Lemma 4.24 of [DS] there exists a constant $C$, independent of $u, \gamma$ and $H$, such that either $d_{\Gamma}(x, u x) \leq C$ or $\ell_{\Gamma}(u) \geq \ell_{\Gamma}^{*}(\gamma H)+$ $\frac{1}{2} d_{\Gamma}(x, u x)-C$.

Thus if $d_{\Gamma}(x, u x)>C$ then $\ell_{\Gamma}^{*}(\gamma H) \leq \ell_{\Gamma}(u)-\frac{1}{2} d_{\Gamma}(x, u x)+C$ As $\ell_{\widehat{\Gamma}}(\hat{\gamma}) \leq$ $\ell_{\hat{\Gamma}}^{*}(\gamma H)+1 \leq \ell_{\Gamma}^{*}(\gamma H)+1$, it follows that $\ell_{\widehat{\Gamma}}(\hat{\gamma}) \leq \ell_{\Gamma}(u)+C+1$.

Otherwise $\ell_{\Gamma}\left(x^{-1} u x\right)=d_{\Gamma}(x, u x) \leq C$. Consider the sequence of elements $x^{-1} u^{n} x \in G$. As $u$ is non-torsion, this is an infinite family of elements. Since $\ell_{\Gamma}$ is a proper length function, there can be at most finitely many elements of $G$ with length not exceeding $C$. Let $M$ be the cardinality of the ball of radius $C$ centered at the identity. Then there is an $N \leq M+1$ such that $\ell_{\Gamma}\left(x^{-1} u^{N} x\right)>C$. Because $u^{N}$ is a non-torsion parabolic element lying in $\gamma H \gamma^{-1}$, one has $\ell_{\Gamma}\left(u^{N}\right) \geq$ $\ell_{\Gamma}^{*}(\gamma H)+\frac{1}{2} d_{\Gamma}\left(x, u^{N} x\right)-C$ so that $\ell_{\widehat{\Gamma}}(\hat{\gamma}) \leq \ell_{\Gamma}\left(u^{N}\right)+C+1$. As $\ell_{\Gamma}\left(u^{N}\right) \leq$ $N \ell_{\Gamma}(u) \leq(M+1) \ell_{\Gamma}(u)$, we have $\ell_{\hat{\Gamma}}(\hat{\gamma}) \leq(M+1) \ell_{\Gamma}(u)+C+1$.

Theorem 2.2.10. Let $G$ be a finitely generated group with generating set $X$ such that $G$ is relatively hyperbolic with respect to $\left\{H_{\lambda}\right\}_{\lambda \in \Lambda}$, each member of which has a $\mathcal{P}$-solvable conjugacy bound. Then $G$ has a $\mathcal{P}$-solvable conjugacy bound.

Proof. Let $\delta$ denote the hyperbolicity constant of the coned-off Cayley graph $\hat{\Gamma}$. As each $H_{\lambda}$ has a $\mathcal{P}$-solvable conjugacy bound, there are polynomials $P_{\lambda}$ such that if $u, v \in H_{\lambda}$ are conjugate in $H_{\lambda}$, then there is a $g \in H_{\lambda}$ with $u^{g}=v$ and $\ell_{H_{\lambda}}(g) \leq P_{\lambda}\left(\ell_{H_{\lambda}}(u)+\ell_{H_{\lambda}}(v)\right)$. As $\left(H_{\lambda}, \ell_{H_{\lambda}}\right) \rightarrow\left(G, \ell_{G}\right)$ is a quasi-isometric embedding, there exists a polynomial $Q_{\lambda}$ such that $\ell_{\Gamma}(g) \leq Q_{\lambda}\left(\ell_{\Gamma}(u)+\ell_{\Gamma}(v)\right)$. If $u$ and $v$ are two elements of $H_{\lambda}$ with $g \in G$ and $u^{g}=v$, then $v \in H_{\lambda}^{g} \cap H_{\lambda}$. If $g \in G \backslash H_{\lambda}$, then Proposition 2.36 [Os] gives $v=1$. Thus if $u$ and $v$ are nontrivial elements of $H_{\lambda}$ which have a conjugator in $G$, then they have a conjugator in $H_{\lambda}$. The $\Gamma(G, X)$-length of the minimal conjugator is thus bounded by the polynomial $Q_{\lambda}$. Moreover, as $\Lambda$ must be finite by Proposition 2.2.1 above, $Q=\sum_{\lambda \in \Lambda} Q_{\lambda}$ is a polynomial which works to bound the conjugator length for every $H_{\lambda}$.

Let $u$ and $v$ be conjugate hyperbolic elements of $G$, and let $L=\ell_{\Gamma}(u)+$ $\ell_{\Gamma}(v)$. According to $[\mathrm{Bu}]$, Lemma 5.11, we have that the $\Gamma$-distance which $g$ 
travels in a coset it penetrates is bounded by $2(L)+10 c(8 L)$, where $c(k)$ is the function given by the BCP property. As shown above, this is bounded by a polynomial in $L$. By Lemma 2.2.8 above, we have $\ell_{\widehat{\Gamma}}(\hat{g}) \leq K_{h}(L)$. As each step of this shortened path corresponds to a $\Gamma$-distance bounded by a polynomial in $L$, it follows that there is an element $g^{\prime} \in G$ conjugating $u$ to $v$ with $\ell_{\Gamma}\left(g^{\prime}\right) \leq$ $\left(2\left(\ell_{\Gamma}(u)+\ell_{\Gamma}(v)\right)+10 c\left(8 \ell_{\Gamma}(u)+8 \ell_{\Gamma}(v)\right)\right) K_{h}\left(\ell_{\Gamma}(u)+\ell_{\Gamma}(v)\right)$. Since $c(k)$ can be bounded by a polynomial in $k$, we see that this expression is bounded by some universal polynomial $Q_{h}\left(\ell_{\Gamma}(u)+\ell_{\Gamma}(v)\right)$.

Let $u$ be a non-torsion parabolic element of $G \backslash H_{\lambda}$ and $v$ an element of $H_{\lambda}$ with $u$ conjugate to $v$. Let $g$ be a conjugator of minimal relative length with $g^{-1} u g=v$. Then $u \in g H_{\lambda} g^{-1}$. By Lemma 2.2.9 we have that $\ell_{\widehat{\Gamma}}(\hat{g}) \leq K_{p}\left(\ell_{\Gamma}(u)\right)$.

Let $g^{\prime}$ be an element with minimal relative length which conjugates $u$ into $H$, and let $h=g^{\prime-1} u g^{\prime}$. By [Bu], Theorem 5.13, the $\Gamma$-length is bounded by $c\left(7 \ell_{\Gamma}(u)+\right.$ $\left.7 \ell_{\Gamma}(v)\right)$. In this case, from our above argument, we have that the element of minimal length conjugating $h$ to $v$ has length bounded by a polynomial in $\ell_{\Gamma}(u)+\ell_{\Gamma}(v)$. Then to bound the length of $g$, we may assume that $g$ has minimal relative length amongst all elements conjugating $u$ into $H$.

Consider the geodesic quadrilateral in $\hat{\Gamma}$ with sides $[e, u],[u, u g],[u g, g]$ and $[e, g]$, where the paths $[e, g]$ and $[u g, g]$ correspond to the shortened path $\hat{g}$. Denote by $\hat{p}$ the path $[u, u g]$ and by $\hat{q}$ the path $[e, g]$. Let $\hat{p}$ travel through the coset $f H$ along the word $k$. By [Bu], Corollary 5.4, if $\hat{q}$ does not penetrate $f H$, then the $\Gamma$-distance of $k$ is at most $2\left(\ell_{\Gamma}(u)+\ell_{\Gamma}(v)\right)+c\left(2 \ell_{\Gamma}(u)+2 \ell_{\Gamma}(v)+1\right)+2 c(2)$. Otherwise $\hat{q}$ also travels through $f H$. Assume that $\hat{q}$ travels through $f H$ along $k^{\prime}$.

If $k=k^{\prime}$ so that $p$ and $q$ travel synchronously through $f H$, let $\gamma_{1}$ be an $H$ geodesic connecting the first point of $p$ in $f H$ to the first point of $q$ in $f H$. Let $\gamma_{2}$ be an $H$-geodesic connecting the last point of $p$ in $f H$ to the last point of $q$ in $f H$. Then $u$ and $v$ are conjugate to $\gamma_{1}$ and $\gamma_{2}$, each of which are in $H$. By the argument in the proof of Theorem 5.12 of [Bu], the relative lengths of $\gamma_{1}$ and $\gamma_{2}$ are each bounded by

$$
\left(7 \ell_{\Gamma}(u)+7 \ell_{\Gamma}(v)+2 \delta+2 \tilde{N}\left(2 \ell_{\Gamma}(u)+2 \ell_{\Gamma}(v)+1\right)\right) .
$$

Moreover, in any coset they penetrate they can travel a $\Gamma$-distance at most

$$
\begin{aligned}
\left(3 c \left(56 \ell_{\Gamma}(u)+56 \ell_{\Gamma}(v)+18 \delta\right.\right. & \left.+16 \tilde{N}\left(2 \ell_{\Gamma}(u)+2 \ell_{\Gamma}(v)+1\right)\right) \\
& \left.+4 \ell_{\Gamma}(u)+4 \ell_{\Gamma}(v)+10 c\left(8 \ell_{\Gamma}(u)+8 \ell_{\Gamma}(v)\right)\right) .
\end{aligned}
$$

Thus their $\Gamma$-lengths are bounded by the product of these two quantities, a polynomial in $\ell_{\Gamma}(u)+\ell_{\Gamma}(v)$. The subpath of $g$ conjugating $\gamma_{1}$ to $\gamma_{2}$ can then be thought of as an element of $H$ conjugating $\gamma_{1}$ to $\gamma_{2}$. Its $\Gamma$-length can then be bounded by a polynomial in $\ell_{\Gamma}\left(\gamma_{1}\right)+\ell_{\Gamma}\left(\gamma_{2}\right)$. Thus the $\Gamma$-distance traveled by $p$ and $q$ through $f H$ is bounded by a universal polynomial in $\ell_{\Gamma}(u)+\ell_{\Gamma}(v)$.

If this is not the case, then $k$ and $k^{\prime}$ are not synchronously in $f H$. Assume that there is a coset $f^{\prime} H$ penetrated by both $\hat{p}$ and $\hat{q}$, such that $\hat{p}$ travels through $f^{\prime} H$ 
along $j, \hat{q}$ travels through $f^{\prime} H$ along $j^{\prime}$ with $\hat{p}$ traveling along $k$ before $j$ and $\hat{q}$ traveling along $j^{\prime}$ before $k^{\prime}$.

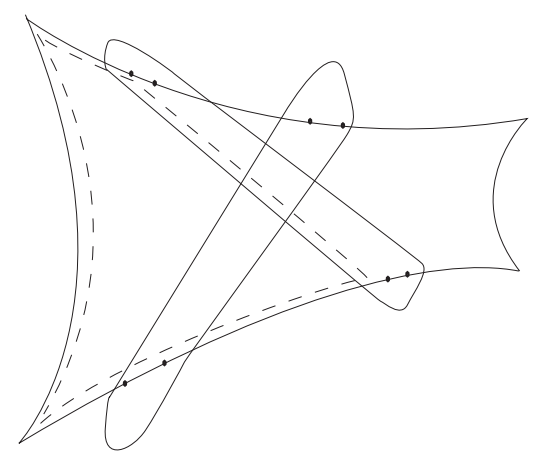

Let $a$ be the first point of $q$ in $f^{\prime} H$, and let $b$ be the first point of $p$ in $f^{\prime} H$. Consider the paths $w_{1}$ and $w_{2}$, where $w_{1}$ is the indicated paths from $e$ to $u$ then to $a$, and $b$ is the path from $e$ to $b$ then to $a$, such that the path from $b$ to $a$ is an $H$-geodesic. As in the 'skew'-coset case of Lemma 5.5 of [Bu], $\hat{w}_{1}$ and $\hat{w}_{2}$ are two $\ell_{\Gamma}(u)+\ell_{\Gamma}(v)+2 \ell_{\widehat{\Gamma}}(\hat{g})$ quasigeodesics, and the $\Gamma$-length of $k$ is bounded by $c\left(\ell_{\Gamma}(u)+\ell_{\Gamma}(v)+2 \ell_{\hat{\Gamma}}(\hat{g})\right)$. As $\ell_{\hat{\Gamma}}(\hat{g})$ is bounded by a polynomial in $\ell_{\Gamma}(u)+\ell_{\Gamma}(v)$, we have that the $\Gamma$-length of $k$ itself is bounded by a polynomial in $\ell_{\Gamma}(u)+\ell_{\Gamma}(v)$.

If this is not the case, then we perform a series of surgeries on the paths $p$ and $q$ as follows. Let $I$ denote the set of $i$ such that $p(i)$ is a point where $p$ enters or exits a coset $f^{\prime} H$ which both $p$ and $q$ penetrate, as well as $i$ such that $q(i)$ is a point where $q$ enters or exits a coset $f^{\prime} H$ which both $p$ and $q$ penetrate. Connect $p(i)$ to $q(i)$ by a $\Gamma$-geodesic, denoted by $\gamma_{i}$, for each $i \in I$. The $\Gamma$-length of each $\gamma_{i}$ is bounded in the same way as $\gamma_{1}$ and $\gamma_{2}$ above. This splits the paths $p$ and $q$ into a series of subpaths which conjugate one $\gamma_{i}$ to the next. We denote by $\gamma_{0}$ and $\gamma_{\infty}$ the paths $u$ and $v$, respectively, and consider 0 and $\infty$ as elements of $I$.

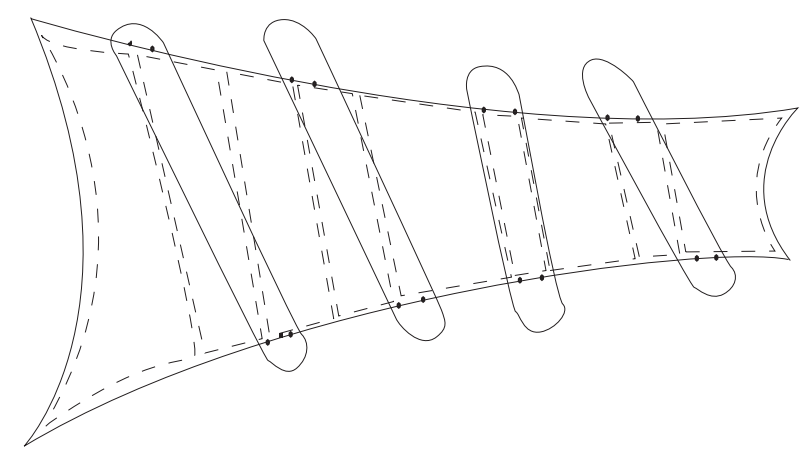

A priori, for each successive $\gamma_{i}, \gamma_{j}$ conjugacy, we have one of the following:

(1) $\gamma_{i}$ and $\gamma_{j}$ are in the same coset. 
(2) $\gamma_{i}$ and $\gamma_{j}$ do not lie in a single common coset, and the subpaths $p^{\prime}$ and $q^{\prime}$ connecting $\gamma_{i}$ to $\gamma_{j}$ do not penetrate a common coset.

(3) $\gamma_{i}$ and $\gamma_{j}$ do not lie in a single common coset, and the subpaths $p^{\prime}$ and $q^{\prime}$ connecting $\gamma_{i}$ to $\gamma_{j}$ do penetrate a common coset.

In each of these cases, the subword of $g$ which conjugates $\gamma_{i}$ to $\gamma_{j}$ can have its length bounded by that of the minimal length conjugator of $\gamma_{i}$ and $\gamma_{j}$. In case (1) the conjugator is a word in $H$ which we have already seen has length bounded by a polynomial in the $\Gamma$-lengths of $\gamma_{i}$ and $\gamma_{j}$. Thus this case is solvable. In case (2) the argument above where the paths $p$ and $q$ do not penetrate a common coset bounds the length of the minimal conjugator by a polynomial in the $\Gamma$-lengths of $\gamma_{i}$ and $\gamma_{j}$. Thus this case is also solvable. In case (3), the subpaths $p^{\prime}$ and $q^{\prime}$ of $p$ and $q$ connecting $\gamma_{i}$ to $\gamma_{j}$ both travel a positive distance through a common coset. Denote this coset by $f H$. By the definition of $I, p(i)$ is the point where $p$ enters $f H$ and $p(j)$ is the point where $p$ exits $f H$, and similarly for $q(i)$ and $q(j)$. Then $\gamma_{i}$ and $\gamma_{j}$ are elements of $H$, and the above argument bounds the length of their conjugator.

As the number of such pairs is bounded by the $\widehat{\Gamma}$-length of $\hat{g}$, which is itself bounded by a polynomial in $\ell_{\Gamma}(u)+\ell_{\Gamma}(v)$, and each $\gamma_{i}$ has length bounded by a polynomial in $\ell_{\Gamma}(u)+\ell_{\Gamma}(v)$. Putting this together with the above arguments, we see that, through any coset which they penetrate, the $\Gamma$-distance which $p$ and $q$ can travel is bounded by a polynomial in $\ell_{\Gamma}(u)+\ell_{\Gamma}(v)$. Thus there is a universal polynomial $U$ such that the element of minimal length conjugating $u$ to $v$ has length bounded by $U\left(\ell_{\Gamma}(u)+\ell_{\Gamma}(v)\right)$.

Let $u$ and $v$ be two conjugate parabolic elements of $G \backslash H_{\lambda}$, which can each be conjugated into $H_{\lambda}$. Assume that $u^{g}=v$, for $g$ a conjugator of minimal length. There is $h \in H_{\lambda}$ such that $u$ is conjugate to $h$, and $v$ is conjugate to $h$, and by Lemma 5.13 of $[\mathrm{Bu}]$ we have $\ell_{\Gamma}(h) \leq c\left(7 \ell_{\Gamma}(u)+7 \ell_{\Gamma}(v)\right)$. By the argument above, there is an element conjugating $u$ to $h$ whose length is bounded by a polynomial in $\ell_{\Gamma}(u)+\ell_{\Gamma}(h)$ and an element conjugating $v$ to $h$ whose length is bounded by a polynomial in $\ell_{\Gamma}(v)+\ell_{\Gamma}(h)$. The product of these two conjugators gives an element of $G$ which conjugates $u$ to $v$, and whose length is bounded by a universal polynomial in $\ell_{\Gamma}(u)+\ell_{\Gamma}(v)$.

Recall that if $L_{1}$ and $L_{2}$ are two length functions on a group $G$, then $L_{1}$ dominates $L_{2}$, provided that there are constants $A$ and $B>0$ such that $L_{2}(g) \leq A\left(L_{1}(g)+1\right)^{B}$ for all $g \in G$. Now $L_{1}$ and $L_{2}$ are equivalent if $L_{1}$ dominates $L_{2}$ and $L_{2}$ dominates $L_{1}$. Viewed as weight functions on $G$, we see that this classical notion of equivalence corresponds to $\mathcal{P}$-equivalence as defined in Section 1.2 above. Thus equivalent length functions on a discrete group yield the same algebra of polynomially bounded functions.

Theorem 2.2.11. Let $G$ be a finitely generated group such that $G$ is relatively hyperbolic with respect to $H_{1}, H_{2}, \ldots, H_{n}$, where each $H_{i}$ is in the class $\mathcal{C}_{\mathcal{P}}(\mathbb{C})$ and 
has a $\mathcal{P}$-solvable conjugacy bound. Then $G$ is in the class $\mathcal{C}_{\mathcal{P}}(\mathbb{C})$. In particular, the $\ell^{1}$-SrBC holds for $G$.

Proof. By Theorem 2.2.10 and the methods of Section 1.4 above,

$$
\begin{aligned}
& \operatorname{HH}_{t}^{*}\left(H_{L}^{1, \infty}(G)\right)_{x} \cong \operatorname{HH}_{t}^{*}\left(H_{L}^{1, \infty}\left(G_{h}\right)\right), \\
& \operatorname{HC}_{t}^{*}\left(H_{L}^{1, \infty}(G)\right)_{x} \cong \operatorname{HC}_{t}^{*}\left(H_{L}^{1, \infty}\left(G_{h}\right)\right) .
\end{aligned}
$$

It remains to show that $G_{h}$ belongs to the class $\mathcal{C}_{\mathcal{P}}(\mathbb{C})$, for each non-torsion element $h$.

Let $h \in G$ be a non-torsion element. If $h$ is hyperbolic, then by Proposition 2.2.2, the embedding $G_{h} \rightarrow G$ is a quasi-isometric embedding. This is precisely the statement that $\left.L_{G}\right|_{G_{h}}$ is equivalent to $L_{G_{h}}$, where $L_{G}$ denotes the standard wordlength function on $G$ (determined by the generating set). We also have $G_{h}$ is virtually cyclic. Thus, $G_{h} \in \mathcal{C}_{\mathcal{P}}(\mathbb{C})$.

Suppose that $h$ is parabolic. Then by Proposition 2.2.3, $G_{h}=x H_{i, x^{-1} h x} x^{-1}$ for some $x \in G$. Thus, $\left.L_{G_{h}}=L_{x\left(H_{i, x}-1 h x\right.}\right) x^{-1}$. As all of the $H_{i}$ are quasi-convex in $G$, the restriction $\left.L_{G}\right|_{x H_{i} x^{-1}}$ is equivalent to $L_{x H_{i} x^{-1}}$. Then $\left.L_{G}\right|_{x H_{i, x^{-1}} x_{x} x^{-1}}$ is equiv-

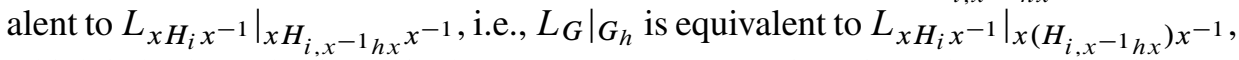
which is by hypothesis equivalent to $L_{x H_{i, h} x^{-1}}$. Conjugating where appropriate we obtain that $\left.L_{G}\right|_{G_{h}}$ is equivalent to $L_{G_{h}}$. Since $G_{h} \cong H_{i, x^{-1} h x}$, while $H_{i}$ is in the class $\mathcal{C}_{\mathcal{P}}(\mathbb{C})$, the theorem is proved.

This verifies Theorem $\mathrm{A}$ in the case $\mathscr{B}=\mathcal{P}$. However, in the more general case when $\mathcal{P} \prec \mathscr{B}$, the argument is the same. In fact, the $\mathscr{B}$-analogue of Theorem 2.2.10 clearly holds whenever $\mathcal{P} \prec \mathscr{B}$, at which point the argument for proving Theorem 2.2.11 carries over with $\mathscr{B}$ replacing $\mathcal{P}$ throughout.

2.3. Combable groups. We begin with the definition of a combing for a discrete group given in [Me1] (this notion is originally due to Thurston [CEHLPT]).

Definition 2.3.1. If $(X, *)$ is a basepointed discrete metric space with distance function $d$, a combing on $X$ consists of a sequence of maps $f_{n}: X \rightarrow X$ satisfying

(i) $f_{0}=*$ and for all $x \in X$ there is an $N$ such that $f_{n}(x)=x$ for all $n \geq N$;

(ii) there exists a constant $C>0$ such that $d\left(f_{n}(x), f_{n}(y)\right) \leq C d(x, y)+1$;

(iii) there exists a constant $S \in \mathbb{N}$ such that $d\left(f_{n}(x), f_{n+1}(x)\right) \leq S$ for all $x \in X$ and $n \in \mathbb{N}$.

For a given $x$, let $J(x)$ be the number of integers $n$ for which $f_{n}(x) \neq f_{n+1}(x)$. The combing is polynomial resp. exponential if $J(x) \leq g(l(x))$ for all $x \in X$, where $g(t)=C^{\prime}(1+t)^{m}$ resp. $C^{\prime} e^{k t}$ for some $C^{\prime}, k \in \mathbb{R}_{+}$and $m \in \mathbb{N}$ (here $l(x):=d(*, x))$. This applies in particular to discrete groups with word-length $(G, L)$, viewed as a basepointed metric space with metric $d\left(g_{1}, g_{2}\right):=L\left(g_{1}^{-1} g_{2}\right)$. 
That $\mathrm{HH}_{t}^{*}\left(H_{\mathcal{B}, L}(G)\right)$ and $\mathrm{HC}_{t}^{*}\left(H_{\mathcal{B}, L}(G)\right)$ should be computable in terms of the Hochschild and cyclic cohomology of $\mathbb{C}[G]$ for combable groups is strongly suggested by the following two results.

Theorem 2.3.2 ([Me1], [Me2], [Og2]). Let $(G, L)$ be a synchronously combable group with polynomial combing function, $L$ the standard word-length function on $G$. Then there is an isomorphism

$$
H_{\mathcal{P}, G}^{*}\left(E G_{\bullet} ; A\right) \stackrel{\cong}{\longrightarrow} H_{G}^{*}\left(E G_{\bullet} ; A\right)
$$

for any p-bounded semi-normed G-module A.

Actually the result of [Me1] only deals with the case that $A$ is the trivial $G$-module $\mathbb{C}$ but also proves the same result when $G$ has an exponential combing function and $\mathcal{P}$ is replaced by $\mathcal{E}$; for more general coefficient modules one needs to use either [Me2] or [Og2] (the definition of a $p$-bounded semi-normed $G$-module is as in [Og2]).

Theorem 2.3.3 ([Me2]). Let $(G, L)$ be as above, and let $P_{*}$ be a bimodule resolution of $\mathbb{C}[G]$ by free $\mathbb{C}[G]$-bimodules. Then $H_{\mathcal{B}, L}(G) \otimes_{\mathbb{C}[G]} P_{*} \otimes_{\mathbb{C}[G]} H_{\mathscr{B}, L}(G)$ is a (bornological) bimodule resolution of $H_{\mathcal{B}, L}(G)$ by free (bornological) $H_{\mathscr{B}, L}(G)$ bimodules $(\mathscr{B}=\mathcal{P}$ or $\mathbb{E})$.

[Note: What is done in [Me2] is much more general; Theorem 2.3.2 only states a special case relevant to our setting].

Proposition 2.3.4. Assume that $(G, L)$ is a group equipped with a polynomial combing in the above sense. Then for each conjugacy class $x$ there are isomorphisms

$$
\begin{aligned}
\operatorname{HH}_{t}^{*}\left(H_{\mathcal{P}, L}(G)\right)_{x} & =\mathrm{HH}_{\mathcal{P}}^{*}(\mathbb{C}[G])_{x} \\
& =H_{\mathcal{P}, G}^{*}\left(E G_{.} ; \operatorname{Hom}_{\mathcal{P}}\left(S_{x}, \mathbb{C}\right)\right) \\
& \cong H_{G}^{*}\left(E G_{\bullet} ; \operatorname{Hom}_{\mathcal{P}}\left(S_{x}, \mathbb{C}\right)\right),
\end{aligned}
$$

where $S_{x}$ is a weighted $G$-set with weight function induced by $L$ and the inclusion into $G, \operatorname{Hom}_{\mathcal{P}}\left(S_{x}, \mathbb{C}\right)$ denotes the subspace of $\operatorname{Hom}\left(S_{x}, \mathbb{C}\right)$ consisting of those maps which are polynomially bounded with respect to the weight function on $S_{x}$, and $H_{\mathcal{P}, G}^{*}(\cdot)$ denotes the cohomology of the cocomplex of $G$-equivariant, polynomially bounded cochains.

Proof. The first equality was verified above. Corollary 1.4.2 gives the identification of $\mathrm{HH}_{\mathcal{P}}^{*}(\mathbb{C}[G])_{x}$ with $H_{\mathcal{P}}^{*}\left(S_{x} \times_{G} E G\right.$. $)=H_{\mathcal{P}, G}^{*}\left(S_{x} \times E G_{\text {. }}\right)$. These last groups can be computed as the cohomology of $\left\{C_{\mathcal{P}, G}^{n}\left(S_{x} \times E G_{.}, \mathbb{C}\right)\right\}_{n \geq 0}=$ $\left\{\operatorname{Hom}_{\mathcal{P}, G}\left(S_{x} \times E G_{n}, \mathbb{C}\right)\right\}_{n \geq 0}$. For each $n$ taking partial adjoints yields an iso-

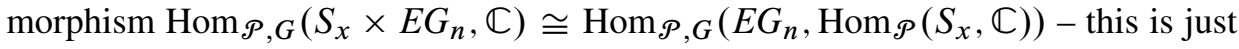
the usual isomorphism on cochains, restricted to polynomially bounded cochains 
(cf. [Og2]); consequently, it commutes with the coboundary maps and extends to an isomorphism of cocomplexes

$$
\begin{aligned}
\left\{\operatorname{Hom}_{\mathcal{P}, G}\left(S_{x} \times E G_{n}, \mathbb{C}\right)\right\}_{n \geq 0} & \cong C_{\mathcal{P}, G}^{*}\left(E G_{.}, \operatorname{Hom}_{\mathcal{P}}\left(S_{x}, \mathbb{C}\right)\right) \\
& :=\left\{\operatorname{Hom}_{\mathcal{P}, G}\left(E G_{n}, \operatorname{Hom}_{\mathcal{P}}\left(S_{x}, \mathbb{C}\right)\right)\right\}_{n \geq 0},
\end{aligned}
$$

which produces the second isomorphism in cohomology. The third follows from Theorem 2.3.2.

Note that $\operatorname{Hom}_{\mathcal{P}}\left(S_{x}, \mathbb{C}\right)$ is dense in $\operatorname{Hom}\left(S_{x}, \mathbb{C}\right)$, and it is reasonable to ask (or even conjecture)

Question/Conjecture 2.3.5. For $(G, L)$ as above, does the inclusion of coefficients $\operatorname{Hom}_{\mathcal{P}}\left(S_{x}, \mathbb{C}\right) \hookrightarrow \operatorname{Hom}\left(S_{x}, \mathbb{C}\right)$ induce an isomorphism

$$
H_{G}^{*}\left(E G_{.} ; \operatorname{Hom}_{\mathcal{P}}\left(S_{x}, \mathbb{C}\right)\right) \stackrel{\cong}{\Longrightarrow} H_{G}^{*}\left(E G_{.} ; \operatorname{Hom}\left(S_{x}, \mathbb{C}\right)\right) ?
$$

If this is the case, then there would be an isomorphism $\operatorname{HH}_{t}^{*}\left(H_{\mathcal{P}, L}(G)\right)_{x} \cong$ $\mathrm{HH}^{*}(\mathbb{C}[G])_{x}$ for each $x$, and so isomorphisms $\operatorname{HC}_{t}^{*}\left(H_{\mathcal{P}, L}(G)\right)_{x} \cong \mathrm{HC}^{*}(\mathbb{C}[G])_{x}$. However, we have not been able to verify these isomorphisms except in certain special cases in which the general theory of Section 1.4 can be applied. The following is an immediate consequence of Proposition 2.3.4.

Corollary 2.3.6. If $\mathbb{Q}$ admits a projective resolution of length $N$ over $\mathbb{Q}[G]$, then $\mathrm{HH}_{t}^{*}\left(H_{\mathcal{P}, L}(G)\right)_{x}=\mathrm{HH}^{*}(\mathbb{C}[G])_{x}=0$ for all $*>N$.

An important subclass of combable groups are the semi-hyperbolic groups defined by Alonso and Bridson in their paper $[\mathrm{AB}]$. We have

Theorem 2.3.7. Let $(G, L)$ be semi-hyperbolic in the sense of $[\mathrm{AB}]$. Then for each conjugacy class $x=\langle h\rangle$, there are isomorphisms

$$
\begin{aligned}
& \operatorname{HH}_{t}^{*}\left(H_{\mathcal{E}, L}(G)\right)_{x} \cong H^{*}\left(B G_{h} ; \mathbb{C}\right)=\mathrm{HH}^{*}(\mathbb{C}[G])_{x}, \\
& \operatorname{HC}_{t}^{*}\left(H_{\mathcal{E}, L}(G)\right)_{x} \cong \mathrm{HC}^{*}(\mathbb{C}[G])_{x} .
\end{aligned}
$$

Proof. By $[\mathrm{AB}]$, we know that quasi-convex subgroups of semi-hyperbolic are again semi-hyperbolic. This applies in particular to the centralizer subgroups $G_{x}$. Consequently, each $G_{x}$ admits a synchronous (linear) combing. By [Me1], $G_{x}$ is $\mathcal{E}$-isocohomogical. Again, by $[\mathrm{AB}]$, we have that $G$ has an $\mathscr{E}$-solvable conjugacy bound. Thus the hypothesis of Theorem 1.4.7 is satisfied for any $\mathscr{B}$ containing $\mathcal{E}$, and the result follows.

Corollary 2.3.8. If $(G, L)$ is semi-hyperbolic and $G$ satisfies the nilpotency condition, then it satisfies the $\mathbb{E}$-nilpotency condition. 
In the absence of an affirmative answer to (2.3.5) above, it would be quite desirable to strengthen Corollary 2.3 .8 by replacing " $\mathcal{E}$-nilpotency" with " $\mathcal{P}$-nilpotency". Certainly the groups $G_{x}$ are $\mathcal{P}$-isocohomological (their combings are even linear). The obstruction lies with the time needed to solve the conjugacy problem. Currently it is not known if the exponential bound on the solution time given in $[\mathrm{AB}]$ is actually a sharp bound in the general case, or whether it can be improved to polynomial time. However, for certain semi-hyperbolic groups, this improved bound is known for certain conjugacy classes. In particular, if $G$ is a mapping class group, then the conjugacy bound is polynomial [MM] when the conjugacy class is pseudo-Anosov. In general the $\mathcal{P}$-solvable conjugacy bound problem is still solvable, but we will address this issue in a future publication.

Finally we remark that the Bass Conjecture for semi-hyperbolic groups was proved by Eckmann [Ec2]

2.4. Hochschild and cyclic homology of $\ell^{1}(G)$. As we have observed, for $\mathcal{B}=$ $\mathscr{B}_{\min }$, the corresponding rapid decay algebra $H_{\mathcal{B}_{\min }, L}(G)$ is equal to $\ell^{1}(G)$, the full $\ell^{1}$-algebra of $G$. More generally, one has an identification of Banach spaces $H_{\mathcal{B}_{\min }, w}(X)=\ell^{1}(X)$ for any weighted space $(X, w)$. Since the class $\mathscr{B}_{\min }$ consists of constant functions, the space $H_{\mathcal{B}_{\min }, w}(X)$ is independent of $w$. For this reason, we will drop $w$ and simply write $\ell^{1}(X)$ for the corresponding space. As in the proof of Lemma 1.3.3, there is, for any two sets $X, X^{\prime}$, a natural isomorphism of Banach spaces

$$
\ell^{1}(X) \hat{\otimes} \ell^{1}\left(X^{\prime}\right) \cong \ell^{1}\left(X \times X^{\prime}\right)
$$

and by Lemma 1.2.4 a natural identification

$$
\ell^{1}(X)^{*} \cong \operatorname{Hom}_{b}(X, \mathbb{C})
$$

between the continuous dual of $\ell^{1}(X)$ and the space of bounded, complex-valued maps on $X$. Consequently, as in Lemma 1.3.6, we have an isomorphism

$$
C_{t}^{*}\left(\ell^{1}(G)\right) \cong C_{b}^{*}\left(N_{\bullet}^{\text {cy }}(G)\right)
$$

between the topological Hochschild cocomplex for $\ell^{1}(G)$ and the cocomplex of bounded cochains on $N_{\bullet}^{\text {cy }}(G)$. Again, this isomorphism preserves the respective decompositions by conjugacy classes, and commutes with the maps in the ConnesGysin sequence. Thus for any conjugacy class $x$ we have an identification

$$
\mathrm{HH}_{t}^{*}\left(\ell^{1}(G)\right)_{x} \cong H_{b}^{*}\left(N_{.}^{\mathrm{cy}}(G)_{x}\right) .
$$

Unlike the case $\mathscr{B} \neq \mathscr{B}_{\min }$, we see that the isomorphism $G_{h} \backslash G \cong S_{x}$ of right $G$ sets (Proposition 1.4.3, Lemma 1.4.4) producing the isomorphism of simplicial sets $\left(\pi_{x}\right)_{.}: G_{h} \backslash G \times_{G} E G$. $\cong S_{x} \times_{G} E G$. will always yield an isomorphism on bounded cochains: $C_{b}^{*}\left(S_{x} \times{ }_{G} E G\right.$. $) \stackrel{\cong}{\longrightarrow} C_{b}^{*}\left(G_{h} \backslash G \times_{G} E G_{\text {. }}\right)$. Given that Proposition 1.4.5 holds without restriction, we conclude 
Corollary 2.4.3. For all discrete groups $G$ and conjugacy classes $x=\langle h\rangle$, there is a natural isomorphism $\mathrm{HH}_{t}^{*}\left(\ell^{1}(G)\right)_{x} \cong H_{b}^{*}\left(B G_{h}\right)$.

We will write $S_{\mathbb{C}}^{*}$ for the $S$-map $S^{*}: \mathrm{HC}^{*}(\mathbb{C}) \rightarrow \mathrm{HC}^{*+2}(\mathbb{C})$. The main result of this section is

Theorem 2.4.4. For all discrete groups $G$ and conjugacy classes $x=\langle h\rangle$ there is an isomorphism

$$
\operatorname{HC}_{t}^{*}\left(\ell^{1}(G)\right)_{x} \cong H_{b}^{*}\left(B G_{h}\right) \otimes \mathrm{HC}^{*}(\mathbb{C}),
$$

where the map $S_{x}^{*}$ on the left identifies with $\operatorname{Id} \otimes S_{\mathbb{C}}^{*}$ on the right.

Proof. The proof is an adaption of the method due to Nistor [Ni1] (see also [Ji2]). For $h \in G$, let $\tilde{L}(G, h)_{.}=(E G$.). Writing $x$ for $\langle h\rangle$, define a projection map $P_{h}: \widetilde{L}(G, h) . \rightarrow N_{.}^{\mathrm{cy}}(G)_{x}$ by $P_{h}\left(g_{0}, \ldots, g_{n}\right)=\left(g_{n}^{-1} h g_{0} \otimes g_{0}^{-1} g_{1} \otimes \cdots \otimes g_{n-1}^{-1} g_{n}\right)$. It not difficult to observe that $P_{h}$ induces an isomorphism of simplicial sets $\bar{P}_{h}: * \times_{G_{h}} \widetilde{L}(G, h) . \stackrel{\cong}{\rightrightarrows} N_{\bullet}^{\text {cy }}(G)_{x}$ and hence an isomorphism of bounded cochain cocomplexes

$$
C_{b}^{*}\left(N_{\bullet}^{\mathrm{cy}}(G)_{x}\right) \stackrel{\cong}{\rightrightarrows} C_{b}^{*}\left(* \times_{G_{h}} \tilde{L}(G, h) .\right) \cong C_{b, G_{h}}^{*}(\widetilde{L}(G, h) .),
$$

where $C_{b, G_{h}}^{*}\left(\tilde{L}(G, h)\right.$.) denotes the subcocomplex of $C_{b}^{*}(\tilde{L}(G, h)$.) consisting of $G_{h}$-equivariant cochains (with coefficients in the trivial $G_{h}$-module $\mathbb{C}$ ). Now consider the map $T$. defined on $\widetilde{L}(G, h)$. by $T_{n+1}\left(g_{0}, \ldots, g_{n}\right)=\left(h g_{n}, g_{0}, \ldots, g_{n-1}\right)$. If $H$ is any subgroup of $G_{h}$ containing $h$, then we see that $T$. descends to a map $\bar{T}$. on $* \times_{H}$ $\widetilde{L}(G, h)$., giving $* \times_{H} \widetilde{L}(G, h)$, the structure of a cyclic simplicial set, and making $\bar{P}_{h}$ an isomorphism of cyclic simplicial sets. Denote the singular resp. cyclic complex of a cyclic simplicial set $X_{\bullet}^{c}$ (with coefficients in $\mathbb{C}$ ) by $C_{*}\left(X_{\bullet}^{c}\right)$ resp. $\mathrm{CC}_{*}\left(X_{\cdot}^{c}\right)$, and the corresponding dual cocomplexes by $C^{*}\left(X_{\bullet}^{c}\right)$ resp. $\mathrm{CC}^{*}\left(X_{\bullet}^{c}\right)$. Clearly an isomorphism $\phi: X_{.}^{c} \stackrel{\cong}{\cong} Y_{0}^{c}$ of cyclic simplicial sets induces an isomorphism on both singular and cyclic (co)complexes. It also induces an isomorphism of bounded cyclic cocomplexes $\mathrm{CC}_{b}^{*}(\phi): \mathrm{CC}_{b}^{*}\left(X_{.}^{c}\right) \stackrel{\cong}{\Longrightarrow} \mathrm{CC}_{b}^{*}\left(Y_{\text {. }}^{c}\right)$ (as we are in char. $0, \mathrm{CC}_{b}^{*}\left(Z_{\text {. }}^{c}\right)$ is quasi-isomorphic to the subcocomplex of $C_{b}^{*}\left(Z^{c}\right)$ consisting of cochains invariant under the cyclic operator). Applying these observations to our situation, we get for each conjugacy class $x=\langle h\rangle$ an isomorphism

$$
\mathrm{CC}_{t}^{*}\left(\ell^{1}(G)\right)_{x} \cong \mathrm{CC}_{b, G_{h}}^{*}(\widetilde{L}(G, h) .) .
$$

Let $C_{h} \subset G_{h}$ denote the cyclic subgroup generated by $h$, and $N_{h}$ the quotient of $G_{h}$ by $C_{h}$. Denote the cohomology groups of $\mathrm{CC}_{b, C_{h}}^{*}\left(\widetilde{L}(G, h)\right.$.) by $\mathrm{HC}_{b, C_{h}}^{*}(\widetilde{L}(G, h)$.$) .$ We claim these groups are Hausdorff, or alternatively, that the reduced and unreduced cohomology groups agree (see Note 1.3.2). In fact, as $C_{h}$ is amenable for all $h$ (it is abelian), the augmentation map $\widetilde{L}(G, h) . \rightarrow *$ induces isomorphisms on bounded 
Hochschild cohomology, and hence also bounded cyclic cohomology. The Hausdorff condition guarantees the existence of a first quadrant spectral sequence strongly converging to $\mathrm{HC}_{b, G_{h}}^{*}\left(\widetilde{L}(G, h)\right.$.) $:=H^{*}\left(\mathrm{CC}_{b, G_{h}}^{*}\left(\widetilde{L}(G, h)\right.\right.$.)) with $E_{2}^{* *}$-term

$$
E_{2}^{* *}=H_{b}^{*}\left(N_{h} ; \operatorname{HC}_{b, C_{h}}^{*}(\tilde{L}(G, h) .)\right)
$$

(compare [No], [Ji1, (3.7)]). As we have just observed, the augmentation map on $\widetilde{L}(G, h)$. yields an isomorphism $\mathrm{HC}_{b, C_{h}}^{*}\left(\widetilde{L}(G, h)\right.$.) $\cong \mathrm{HC}^{*}(\mathbb{C})$, and hence an isomorphism

$$
E_{2}^{* *}=H_{b}^{*}\left(N_{h} ; \mathrm{HC}_{b, C_{h}}^{*}(\widetilde{L}(G, h) .)\right) \stackrel{\cong}{\longrightarrow} H_{b}^{*}\left(N_{h} ; \mathrm{HC}^{*}(\mathbb{C})\right)
$$

of $E_{2}^{* *}$-terms. The spectral sequence on the right converges to $H_{b}^{*}\left(N_{h}\right) \otimes \mathrm{HC}^{*}(\mathbb{C})$; however by the Serre spectral sequence in bounded cohomology associated to the exact sequence $C_{h} \longrightarrow \rightarrow G_{h} \stackrel{\text { proj }_{h}}{\longrightarrow} N_{h}$ (loc. cit.), we have $H_{b}^{*}\left(N_{h}\right) \stackrel{\text { proj }_{h}}{\cong} H_{b}^{*}\left(G_{h}\right)$, completing the proof.

Thus, although computable, the non-elliptic summands of the topological cyclic cohomology groups of $\ell^{1}(G)$ will never have a nilpotent $S$-operator, and so are not useful in verifying the $\ell^{1}$-Bass conjecture by the methods used in this paper. Note that if $G$ itself is amenable, then each $G_{x}$ is as well, and Theorem 2.4.4 produces an isomorphism between $\operatorname{HC}_{t}^{*}\left(\ell^{1}(G)\right)$ and the $\ell^{1}$-completion of the vector space $\bigoplus_{x \in\langle G\rangle} \mathrm{HC}^{*}(\mathbb{C})$. 


\section{Appendix by C. Ogle. The Baum-Connes assembly map and the generalized Bass Conjecture}

In the early 1980s, P. Baum and A. Connes defined an assembly map

$$
\mathcal{A}_{*}^{G, a}: \mathrm{KK}_{*}^{G}(C(\underline{E} G), \mathbb{C}) \rightarrow K_{*}^{t}\left(C_{r}^{*}(G)\right)
$$

where $G$ denotes a locally compact group, $E G$ the classifying space for proper $G$ actions, $C(E G)$ the $G$-algebra of complex-valued functions on $E G$ vanishing at infinity, and $\mathrm{KK}_{*}^{G}(C(\underline{E} G), \mathbb{C})$ the $G$-equivariant KK-groups of $(\underline{E} G)$ with coefficients in $\mathbb{C}$, while $K_{*}^{t}\left(C_{r}^{*}(G)\right)$ represents the topological $K$-groups of the reduced $C^{*}$-algebra of $G .^{\dagger}$ The original details of this map appeared (a few years later) in [BC1] and [BC2], with further elaborations in [BCH]. As shown in [BC3], when $G$ is discrete the left-hand side admits a Chern character which may be represented as

$$
\mathrm{ch}_{*}^{B C}(G): \mathrm{KK}_{*}^{G}(C(\underline{E} G), \mathbb{C}) \rightarrow \bigoplus_{x \in \operatorname{fin}(\langle G\rangle)} H_{*}\left(B G_{x} ; \mathbb{C}\right) \otimes \operatorname{HPer}_{*}(\mathbb{C})
$$

where fin $(\langle G\rangle)$ is the set of conjugacy classes of $G$ corresponding to elements of finite order, $G_{x}$ the centralizer of $g$ in $G$ where $x=\langle g\rangle$, and $\operatorname{HPer}_{*}(\mathbb{C})$ the periodic cyclic homology of $\mathbb{C}$. Note that $H_{*}(B H ; \mathbb{C}) \otimes \operatorname{HPer}_{*}(\mathbb{C})$ are simply the 2-periodized complex homology groups of $B H$, and (via the classical Atiyah-Hirzebruch Chern character) can be alternatively viewed as the complexified $K$-homology groups of $B H$. Upon complexification, the map $\mathrm{ch}_{*}^{B C}(G)$ is an isomorphism. The original construction of Baum and Connes $\mathcal{A}_{*}^{G, a}$ was analytical. Motivated by the need to construct a homotopical analogue to their map, we constructed an assembly map in $[\mathrm{Og} 1]$ which we will denote here as

$$
\mathcal{A}_{*}^{G, h} \otimes \mathbb{C}: H_{*}\left(\coprod_{x \in \operatorname{fin}(\langle G\rangle)} B G_{x} ; \mathbf{K}(\mathbb{C})\right) \otimes \mathbb{C} \rightarrow K_{*}^{t}\left(C_{r}^{*}(G)\right) \otimes \mathbb{C},
$$

where $\mathbf{K}(\mathbb{C})$ denotes the 2-periodic topological K-theory spectrum of $\mathbb{C}$. The construction of this map amounted to an extension of the classical assembly map constructed in $[\mathrm{L}]$ which was designed to take into account the contribution coming from the conjugacy classes of finite order.

As before, let $\mathbb{C}[G]$ denote the complex group algebra topologized with the fine topology. To this algebra we associate the space

$$
K^{f}(\mathbb{C}[G]):=\left|[q] \mapsto K_{0}\left(S_{q}(\mathbb{C}[G])\right) \times B \mathrm{BL}^{+}\left(S_{q}(\mathbb{C}[G])\right)\right|
$$

where $\left\{S_{q}(X)\right\}$ denotes the total singular complex of the topological space $X$, and $\left|[q] \mapsto Y_{q}\right|$ the geometric realization of the simplicial set $Y$. This space has a

\footnotetext{
${ }^{\dagger}$ For non-cocompact $\underline{E} G$, the equivariant $\mathrm{KK}$-groups $\mathrm{KK}_{*}^{G}(C(\underline{E} G), \mathbb{C})$ are defined as a colimit over the directed set $\operatorname{Co}_{G}(\underline{E} \bar{G})$ of $G$-cocompact subsets of $\underline{E} G$, with the relation given by inclusion:

$$
\mathrm{KK}_{*}^{G}(C(\underline{E} G), \mathbb{C}):=\operatorname{colim}_{\mathrm{Co} G}(\underline{E} G) \mathrm{KK}_{*}^{G}(C(X), \mathbb{C}) .
$$
}


natural infinite-loop structure coming from the standard (non-connective) QuillenGrayson-Wagoner delooping of the spaces on the right, and the associated spectrum is a module over the spectrum delooping of $K^{f}(\mathbb{C})$. In particular, there is a Bott element $\beta \in \pi_{2}\left(K^{f}(\mathbb{C})\right.$ (corresponding to the usual Bott element in $K_{2}^{t}(\mathbb{C})$, and multiplication with this element induces a map

$$
\beta_{*}: \pi_{*}\left(K^{f}(\mathbb{C}[G])\right) \rightarrow \pi_{*+2}\left(K^{f}(\mathbb{C}[G])\right) .
$$

For the purpose of this appendix, we define the topological K-theory of $\mathbb{C}[G]$ as

$$
K_{*}^{t}(\mathbb{C}[G]):=\pi_{*}\left(K^{f}(\mathbb{C}[G])\right)\left[\beta^{-1}\right] .
$$

The two essential features of $\mathcal{A}_{*}^{G, h} \otimes \mathbb{C}$, shown in [Og1], were (i) it factors through $K_{*}^{t}(\mathbb{C}[G]) \otimes \mathbb{C}$, and (ii) the composition of $\mathcal{A}_{*}^{G, h} \otimes \mathbb{C}$ with the complexified ChernConnes-Karoubi-Tillmann character $\mathrm{ch}_{*}^{\mathrm{CK}}: K_{*}(\mathbb{C}[G]) \otimes \mathbb{C} \rightarrow \mathrm{HC}_{*}(\mathbb{C}[G])$ was effectively computable (see below). What we did not do in [Og1] was to show that $\mathcal{A}_{*}^{G, a} \otimes \mathbb{C}$ and $\mathcal{A}_{*}^{G, h} \otimes \mathbb{C}$ agree. Since this initial work, there have been numerous extensions and reformulations of the Baum-Connes assembly map, as well as of the original Baum-Connes conjecture, which states that the map in $(0.1)$ is an isomorphism. These extensions typically are included under the umbrella term "Isomorphism Conjecture" (formulated for both algebraic and topological K-theory; cf. [DL], [FJ], [LR]). Thanks to [HP] we now know that the different formulations of these assembly maps (e.g., homotopy-theoretic vs. analytical) agree.

Abbreviating $\mathrm{KK}_{*}^{G}(C(\underline{E} G), \mathbb{C})$ as $K_{*}^{G}(\underline{E G})$ (read: the equivariant $K$-homology of the proper $G$-space $\underline{E} G)$, our main result is

Theorem A.1. There is a commuting diagram

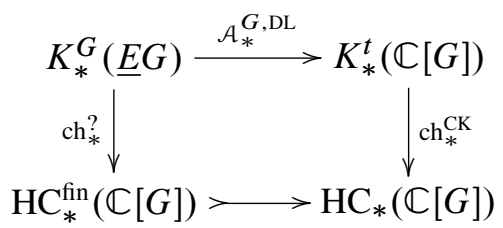

where ${ }^{\text {fin }} \mathrm{HC}_{*}(\mathbb{C}[G]):=\bigoplus_{x \in \operatorname{fin}(\langle G\rangle)} \mathrm{HC}_{*}(\mathbb{C}[G])_{x} \cong \oplus_{x \in \operatorname{fin}(\langle G\rangle)} H\left(B G_{x} ; \mathbb{C}\right) \otimes$ $\mathrm{HC}_{*}(\mathbb{C})$ is the elliptic summand of $\mathrm{HC}_{*}(\mathbb{C}[G]), \mathcal{A}_{*}^{G, \mathrm{DL}}$ is the homotopically defined assembly map of [DL], the lower horizontal map is the obvious inclusion, and the Chern character $\mathrm{ch}_{*}^{?}$ becomes an isomorphism upon complexification for $* \geq 0$.

Let $\beta$ denote a bounding class, $(G, L)$ a discrete group equipped with a wordlength, and $H_{\beta, L}(G)$ the rapid decay algebra associated with this data. We write $K_{*}^{t}\left(H_{\beta, L}(G)\right)$ for the Bott-periodic topological K-theory of the topological algebra $H_{\beta, L}(G)$. The Baum-Connes assembly map for $H_{\beta, L}(G)$ is defined to be the composition

$$
\mathcal{A}_{*}^{G, \beta}: K_{*}^{G}(\underline{E} G) \stackrel{\mathcal{A}_{*}^{G, \mathrm{DL}}}{\longrightarrow} K_{*}^{t}(\mathbb{C}[G]) \rightarrow K_{*}^{t}\left(H_{\beta, L}(G)\right),
$$


where the second map is induced by the natural inclusion $\mathbb{C}[G] \hookrightarrow H_{\beta, L}(G)$. In the introduction, we conjectured that the image of $\mathrm{ch}_{*}: K^{t}\left(H_{\beta, L}(G)\right) \rightarrow \mathrm{HC}_{*}^{t}\left(H_{\beta, L}(G)\right)$ lies in the elliptic summand fin $\mathrm{HC}_{*}^{t}\left(H_{\beta, L}(G)\right.$ ) (conjecture $\beta$-SrBC). As the inclusion $\mathbb{C}[G] \hookrightarrow H_{\beta, L}(G)$ sends ${ }^{\text {fin }} \mathrm{HC}_{*}(\mathbb{C}[G])$ to ${ }^{\text {fin }} \mathrm{HC}_{*}^{t}\left(H_{\beta, L}(G)\right)$, naturality of the Chern character $\mathrm{ch}_{*}^{\mathrm{CK}}$ and Theorem 1 implies

Corollary A.2. If $\mathcal{A}_{*}^{G, \beta}$ is rationally surjective, then $\beta$-SrBC is true.

As going down and then across is rationally injective, we also have (see [O1])

Corollary A.3. The assembly map $\mathcal{A}_{*}^{G, D L} \otimes \mathbb{Q}$ is injective for all discrete groups $G$.

We do not claim any great originality in this paper. In fact, Theorem A.1, although not officially appearing in print before this time, has been a "folk-theorem" known to experts for many years. The connection between the Baum-Connes Conjecture (more precisely a then-hypothetical Baum-Connes-type Conjecture for $\mathbb{C}[G]$ ) and the stronger Bass Conjecture for $\mathbb{C}[G]$ discussed in the introduction was noted by the author in [O4].

There is some overlap of this paper with the results presented in [Ji]. A special case of Theorem 1 (for $*=0$ and $\mathbb{C}[G]$ replaced by the $\ell^{1}$-algebra $\ell^{1}(G)$ ) appeared as the main result of $[\mathrm{BCM}]$.

\section{Proof of Theorem A.1}

We use the notation $F_{*}^{\text {fin }}(\mathbb{C}[G])$ to denote the elliptic summand $\bigoplus_{x \in \operatorname{fin}(\langle G\rangle)} F_{*}(\mathbb{C}[G])_{x}$ of $F_{*}(\mathbb{C}[G])$ where $F_{*}(\cdot)=\mathrm{HH}_{*}(\cdot), \mathrm{HN}_{*}(\cdot), \mathrm{HC}_{*}(\cdot)$ or HPer $(\cdot)$. To maximize consistency with [LR], we write $\mathbf{S}$ for the (unreduced) suspension spectrum of the zero-sphere $S^{0}, \mathbf{H N}(R)$ resp. $\mathbf{H H}(R)$ the Eilenberg-MacLane spectrum whose homotopy groups are the negative cyclic resp. Hochschild homology groups of the discrete ring $R$, and $\mathbf{K}^{a}(R)$ the non-connective algebraic K-theory spectrum of $R$, with $K_{*}^{a}(R)$ representing its homotopy groups. By [LR], diagram 1.6, there is a commuting diagram

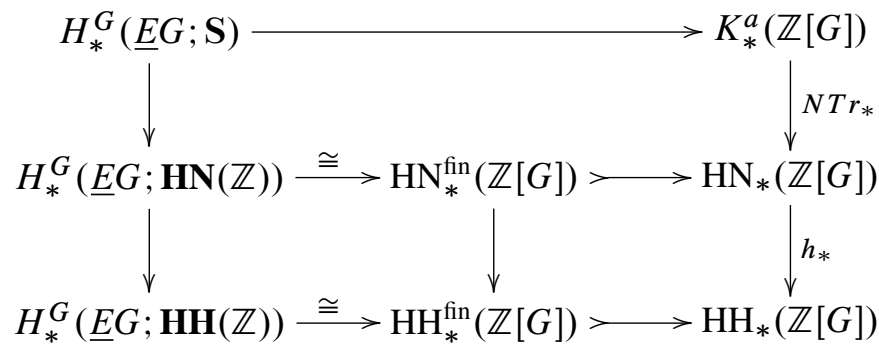

where the top horizontal map is the composition

$$
H_{*}^{G}(\underline{E} G ; \mathbf{S}) \rightarrow H_{*}^{G}\left(\underline{E} G ; \mathbf{K}^{a}(\mathbb{Z})\right) \stackrel{\mathcal{A}^{G, \mathrm{DL}}}{\longrightarrow} K_{*}(\mathbb{Z}[G])
$$


referred to as the restricted assembly map for the algebraic $K$-groups of $\mathbb{Z}[G]$. The other two horizontal maps are the assembly maps for negative cyclic and Hochschild homology respectively. The upper left-hand map is induced by the map from the sphere spectrum to the Eilenberg-MacLane spectrum HN, which may be expressed as the composition of spectra $\mathbf{S} \rightarrow \mathbf{K}^{a}(\mathbb{Z}) \rightarrow \mathbf{H N}$. By [LR], the composition on the left is a rational equivalence.

Let $\mathbb{C}^{\delta}$ denote the complex numbers $\mathbb{C}$ equipped with the discrete topology. Tensoring with $\mathbb{C}$ and combined with the inclusion of group algebras $\mathbb{Z}[G] \hookrightarrow \mathbb{C}^{\delta}[G]$, (A.1.1) yields the commuting diagram

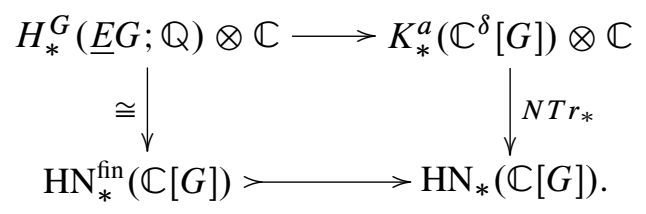

Next we consider the transformation from algebraic to topological K-theory, induced by the map of group algebras $\mathbb{C}^{\delta}[G] \rightarrow \mathbb{C}[G]$ which is the identity on elements. By the results of $[\mathrm{CK}],[\mathrm{W}]$ and $[\mathrm{T}]$, there is a commuting diagram

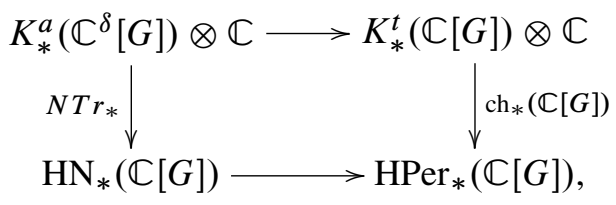

where $\operatorname{ch}_{*}(\mathbb{C}[G])$ is the Connes-Karoubi Chern character for the fine topological algebra $\mathbb{C}[G]$, and the bottom map is the transformation from negative cyclic to periodic cyclic homology.

We can now consider our main diagram

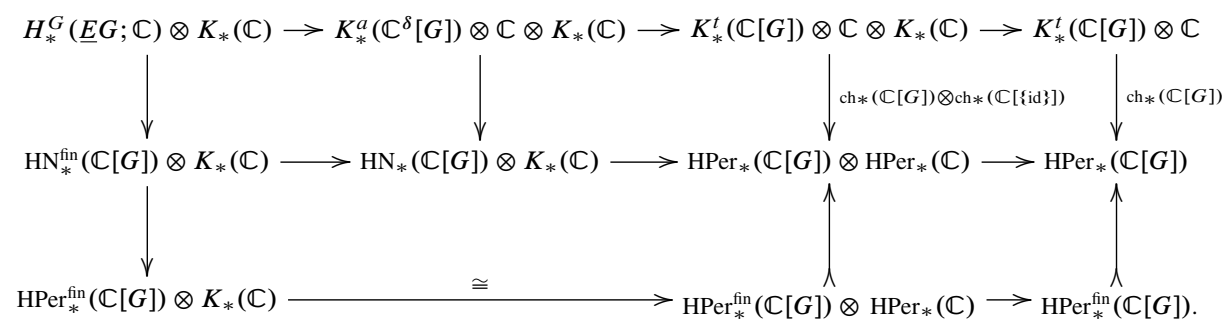

(A.1.4)

The top left square commutes by (A.1.2), and the middle top square commutes by (A.1.3). The upper right square commutes by virtue of the fact that the ConnesKaroubi-Chern character is a homomorphism of graded modules, which maps the $K_{*}^{t}(\mathbb{C})$-module $K_{*}^{t}(\mathbb{C}[G])$ to the $\operatorname{HPer}_{*}(\mathbb{C})$-module $\operatorname{HPer}_{*}(\mathbb{C}[G])$, with the map of base rings induced by isomorphism $\mathrm{ch}_{*}(\mathbb{C}[\{\mathrm{id}\}]): K_{*}^{t}(\mathbb{C}) \otimes \mathbb{C} \cong \operatorname{HPer}_{*}(\mathbb{C})$. The lower left square commutes trivially, while the lower right commutes by the naturality 
of the inclusion $\operatorname{HPer}_{*}^{\mathrm{fin}}(\mathbb{C}[G]) \hookrightarrow \operatorname{HPer}_{*}(\mathbb{C}[G])$ with respect to the module structure over $\mathrm{HPer}_{*}(\mathbb{C})$. Summarizing, we get a commuting diagram

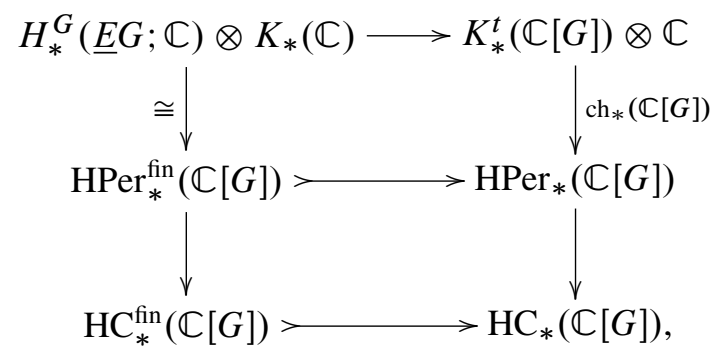

where the top map is the complexified Davis-Lück assembly map for topological group algebra $\mathbb{C}[G]$, and the bottom square is induced by the transformation $\operatorname{HPer}_{*}(\cdot) \rightarrow \mathrm{HC}_{*}(\cdot)$ (which respects the summand decomposition indexed on conjugacy classes). Restricted the elliptic summand yields the map $\operatorname{HPer}_{*}^{\text {fin }}(\mathbb{C}[G]) \rightarrow$ $\mathrm{HC}_{*}^{\mathrm{fin}}(\mathbb{C}[G])$ which is an isomorphism for $* \geq 0$, implying the result stated in Theorem A.1.

\section{References}

[AB] J. M. Alonso and M. R. Bridson, Semihyperbolic groups. Proc. London Math. Soc. (3) 70 (1995), 56-114. Zbl 0823.20035 MR 1300841

[Ba] H. Bass, Euler characteristics and characters of discrete groups. Invent. Math. 35 (1976), 155-196. Zbl 0365.20008 MR 0432781

[BC2] P. Baum and A. Connes, $K$-theory for discrete groups. In Operator algebras and applications, Vol. 1, London Math. Soc. Lecture Note Ser. 135, Cambridge Univ. Press, Cambridge 1988, 1-20. Zbl 0685.46041 MR 0996437

[BC3] P. Baum and A. Connes, Chern character for discrete groups. In A fête of topology, Academic Press, Boston 1988, 163-232. Zbl 0656.55005 MR 0928402

[BC1] P. Baum and A. Connes, Geometric $K$-theory for Lie groups and foliations. Enseign. Math. (2) 46 (2000), 3-42. Zbl 0985.46042 MR 1769535

$[\mathrm{BCH}]$ P. Baum, A. Connes, and N. Higson, Classifying space for proper actions and $K$-theory of group $C^{*}$-algebras. In $C^{*}$-algebras: 1943-1993 (San Antonio, TX, 1993), Contemp. Math. 167, Amer. Math. Soc., Providence, RI, 1994, 241-291. Zbl 0830.46061 MR 1292018

[BCM] A. J. Berrick, I. Chatterji, and G. Mislin, From acyclic groups to the Bass conjecture for amenable groups. Math. Ann. 329 (2004), 597-621. Zbl 1135.20035 MR 2076678

[B1] N. Blackburn, Conjugacy in nilpotent groups. Proc. Amer. Math. Soc. 16 (1965), 143-148. Zbl 0127.01302 MR 0172925 
[BH] M. R. Bridson and A. Haefliger, Metric spaces of non-positive curvature. Grundlehren Math. Wiss. 319, Springer-Verlag, Berlin 1999. Zbl 0988.53001 MR 1744486

[Bu] I. Bumagin, The conjugacy problem for relatively hyperbolic groups. Algebr. Geom. Topol. 4 (2004), 1013-1040. Zbl 1111.20035 MR 2100689

[Bur] D. Burghelea, The cyclic homology of the group rings. Comment. Math. Helv. 60 (1985), 354-365. Zbl 0595.16022 MR 814144

[Co1] A. Connes, Non-commutative differential geometry. Inst. Hautes Études Sci. Publ. Math. 62 (1985), 41-144. Zbl 0592.46056 MR 0823176

[Co2] A. Connes A. Connes, Noncommutative geometry. Academic Press, San Diego 1994. Zbl 0818.46076 MR 1303779

[CK] A. Connes and M. Karoubi, Caractère multiplicatif d'un module de Fredholm. $C$. R. Acad. Sci. Paris Sér. I Math. 299 (1984), 963-968. Zbl 0596.46044 MR 774679

[DL] J.F. Davis and W. Lück, Spaces over a category and assembly maps in isomorphism conjectures in $K$ - and $L$-theory. $K$-Theory 15 (1998), 201-252. Zbl 0921.19003 MR 1659969

[DS] C. Druţu and M. Sapir, Groups acting on tree-graded spaces and splittings of relatively hyperbolic groups. Adv. Math. 217 (2008), 1313-1367. Zbl 1138.20024 MR 2383901

[Ec1] B. Eckmann, Cyclic homology of groups and the Bass conjecture. Comment. Math. Helv. 61 (1986), 193-202. Zbl 0613.20034 MR 856086

[Ec2] B. Eckmann, Idempotents in a complex group algebra, projective modules, and the von Neumann algebra. Arch. Math. (Basel) 76 (2001), 241-249. Zbl 0992.16020 MR 1825003

[Em] I. Emmanouil, On a class of groups satisfying Bass' conjecture. Invent. Math. 132 (1998), 307-330. Zbl 0913.20002 MR 1621420

[CEHLPT] D. B. A. Epstein, J. W. Cannon, D. F. Holt, S. V. F. Levy, M. S. Paterson, W. P. Thurston, Word processing in groups. Jones and Bartlett Publishers, Boston 1992. Zbl 0764.20017 MR 1161694

[Fa] B. Farb, Relatively hyperbolic groups. Geom. Funct. Anal. 8 (1998), 810-840. Zbl 0985.20027 MR 1650094

[FJ] F. T. Farrell and L. E. Jones, Isomorphism conjectures in algebraic $K$-theory. $J$. Amer. Math. Soc. 6 (1993), 249-297. Zbl 0798.57018 MR 1179537

[Gr] M. Gromov, Hyperbolic groups. In Essays in group theory, Math. Sci. Res. Inst. Publ. 8, Springer-Verlag, New York 1987, 75-263. Zbl 0634.20015 MR 0919829

[HP] I. Hambleton and E. Petersen, Identifying assembly maps in $K$ - and $L$-theory. Math. Ann. 328 (2004), 27-57. Zbl 1051.19002 MR 2030369

[Ha] A. Hattori, Rank element of a projective module. Nagoya Math. J. 25 (1965), 113-120. Zbl 0142.28001 MR 0175950

[HK] N. Higson and G. Kasparov, $E$-theory and $K K$-theory for groups which act properly and isometrically on Hilbert space. Invent. Math. 144 (2001), 23-74. Zbl 0988.19003 MR 1821144 
[HN1] H. Hogbe-Nlend, Complétion, tenseurs et nucléarité en bornologie. J. Math. Pures Appl. (9) 49 (1970), 193-288. Zbl 0199.18001 MR 0279557

[HN2] H. Hogbe-Nlend, Bornologies and functional analysis. North-Holland Math. Stud. 26, North-Holland Publishing Co., Amsterdam 1977. Zbl 0359.46004 MR 0500064

[Ji1] R. Ji, Smooth dense subalgebras of reduced group $C^{*}$-algebras, Schwartz cohomology of groups, and cyclic cohomology. J. Funct. Anal. 107 (1992), 1-33. Zbl 0787.46043 MR 1165864

[Ji2] R. Ji, A module structure on cyclic cohomology of group graded algebras. $K$ Theory 7 (1993), 369-399. Zbl 0799.19002 MR 1246282

[Ji3] R. Ji, Nilpotency of Connes' periodicity operator and the idempotent conjectures. K-Theory 9 (1995), 59-76. Zbl 0826.46063 MR 1340840

[Ji] R. Ji, Bivariant Chern character and the analog Baum-Connes map. In Operator algebras and operator theory (Shanghai, 1997), Contemp. Math. 228, Amer. Math. Soc., Providence, RI, 1998, 133-149. Zbl 0930.46056 MR 1667659

[Jo1] P. Jolissaint, Les fonctions à décroissance rapide dans les $C^{*}$-algèbres résduites de groupes. PhD Thesis, Univ. of Geneva, 1987.

[Jo2] P. Jolissaint, $K$-theory of reduced $C^{*}$-algebras and rapidly decreasing functions on groups. K-Theory 2 (1989), 723-735. Zbl 0692.46062 MR 1010979

[L] J.-L. Loday, $K$-théorie algébrique et représentations de groupes. Ann. Sci. École Norm. Sup. (4) 9 (1976), 309-377. Zbl 0362.18014 MR 0447373

[Lo] J.-L. Loday, Cyclic homology. 2nd ed., Grundlehren Math. Wiss. 301, SpringerVerlag, Berlin 1998. Zbl 0885.18007 MR 1600246

[LR] W. Lück and H. Reich, Detecting $K$-theory by cyclic homology. Proc. Lond. Math. Soc. (3) 93 (2006), 593-634. Zbl 1116.19002 MR 2266961

[Ma] J. Maher, Random walks on the mapping class group. Preprint 2006. arXiv:math/0604433

[MM] H. A. Masur and Y. N. Minsky, Geometry of the complex of curves II. Hierarchical structure. Geom. Funct. Anal. 10 (2000), 902-974. Zbl 0972.32011 MR 1791145

[Me3] R. Meyer, Bornological versus topological analysis in metrizable spaces. In $\mathrm{Ba}$ nach algebras and their applications, Contemp. Math. 363, Amer. Math. Soc., Providence, RI, 2004, 249-278. Zbl 1081.46004 MR 2097966

[Me2] R. Meyer Embeddings of derived categories of bornological modules. Preprint 2004. arXiv:math/0410596

[Me1] R. Meyer, Combable groups have group cohomology of polynomial growth. Quart. J. Math. 57 (2006), 241-261. Zbl 1167.20028 MR 2237601

[MF] A. S. Miščenko and A. T. Fomenko, The index of elliptic operators over $C^{*}$ algebras. Izv. Akad. Nauk SSSR Ser. Mat. 43 (1979), 831-859; English transl. Math. USSR-Izv. 15 (1980), 87-112. Zbl 0416.46052 MR 548506

[MV] G. Mislin and A. Valette, Proper group actions and the Baum-Connes conjecture. Birkhäuser Verlag, Basel 2003. Zbl 1028.46001 MR 2027168 
[Ni1] V. Nistor, Group cohomology and the cyclic cohomology of crossed products. Invent. Math. 99 (1990), 411-424. Zbl 0692.46065 MR 1031908

[Ni2] V. Nistor, A bivariant Chern-Connes character. Ann. of Math. (2) 138 (1993), 555590. Zbl 0798.46051 MR 1247993

[No] G. A. Noskov, The Hochschild-Serre spectral sequence for bounded cohomology. In Proceedings of the International Conference on Algebra, Part 1 (Novosibirsk, 1989), Contemp. Math. 131, Amer. Math. Soc., Providence, RI, 1992, 613-629. Zbl 0773.20020 MR 1175809

[Og1] C. Ogle, Assembly maps, $K$-theory, and hyperbolic groups. K-Theory 6 (1992), 235-265. Zbl 0776.19004 MR 1189277

[Og2] C. Ogle, Polynomially bounded cohomology and discrete groups. J. Pure Appl. Algebra 195 (2005), 173-209. Zbl 1071.18007 MR 2109110

[Og3] C. Ogle, $B$-bounded cohomology and discrete groups. In preparation.

[Og4] C. Ogle, Private communication to P. Baum and A. Connes, 1989.

[Os] D. V. Osin, Relatively hyperbolic groups: intrinsic geometry, algebraic properties, and algorithmic problems. Mem. Amer. Math. Soc. 179 (2006), no. 843. Zbl 1093.20025 MR 2182268

[St] J. Stallings, Centerless groups - an algebraic formulation of Gottlieb's theorem. Topology 4 (1965), 129-134. Zbl 0201.36001 MR 0202807

[Ta] J. L. Taylor, Homology and cohomology for topological algebras. Adv. Math. 9 (1972), 137-182. Zbl 0271.46040 MR 0328624

[Ti] U. Tillmann, $K$-theory of fine topological algebras, Chern character, and assembly. K-Theory 6 (1992), 57-86. Zbl 0776.19005 MR 1186774

[W] C. A. Weibel, Nil $K$-theory maps to cyclic homology. Trans. Amer. Math. Soc. 303 (1987), 541-558. Zbl 0627.18005 MR 902784

Received July 26, 2007; revised December 19, 2008

R. Ji, Department of Mathematical Sciences, IUPUI, 402 N. Blackford Street, Indianapolis, IN 46202-3267, U.S.A.

E-mail: ronji@math.iupui.edu

C. Ogle, Department of Mathematics, The Ohio State University, 231 West 18th Avenue,

Columbus, OH 43210-1174, U.S.A.

E-mail: ogle@math.osu.edu

B. Ramsey, Department of Mathematical Sciences, IUPUI, 402 N. Blackford Street, Indianapolis, IN 46202-3267, U.S.A.

E-mail: bramsey@math.iupui.edu 